\title{
VULNERABILIDAD SOCIOAMBIENTAL Y RESILIENCIA EN DOS BARRIOS DEL BORDE URBANO DE LA CIUDAD DE MAR DEL PLATA: APORTES PARA LA GESTIÓN LOCAL
}

Rótolo, Miguel ${ }^{\mathrm{I}}$; Zulaica, Laura ${ }^{2}$

1. Instituto del Hábitat y del Ambiente, Facultad de Arquitectura, Urbanismo y Diseño, Universidad Nacional de Mar del Plata - ProMeBa, Municipio de General Pueyrredon, Mar del Plata, Argentina mcrotolo@gmail.com

2. Consejo Nacional de Investigaciones Científicas y Técnicas (CONICET) - Instituto del Hábitat y del Ambiente, Facultad de Arquitectura, Urbanismo y Diseño, Universidad Nacional de Mar del Plata ProMeBa, Municipio de General Pueyrredon, Mar del Plata, Argentina, laurazulaica@conicet.gov.ar

\section{RESUMEN}

La pandemia por COVID-19 y las medidas de Aislamiento y Distanciamiento Social Preventivo y Obligatorio transformaron las actividades básicas de producción y reproducción de la población. Estos hechos impactaron en las dinámicas sociales y económicas de las ciudades, generando y profundizando desigualdades. Mar del Plata, Argentina, fue una de las ciudades más afectadas en la provincia de Buenos Aires. Los efectos han tenido alcances territoriales diferenciados, siendo más significativos en la periferia urbana. Entre los barrios de estas áreas, se destacan Las Heras y Parque Palermo, enmarcados en el Programa de Mejoramiento de Barrios (ProMeBa) financiado por Banco Interamericano de Desarrollo.

El presente trabajo analiza aspectos relevantes de la vulnerabilidad socioambiental y de la resiliencia comunitaria en el contexto de pande-

\section{ABSTRACT}

The COVID-19 pandemic and the Social Preventive and Mandatory Isolation and Distancing measures transformed the population's basic production and reproduction activities. These events had an impact on the social and economic dynamics of the cities, generating and deepening inequalities. Mar del Plata, Argentina, was one of the most affected cities in the Buenos Aires province. The effects have had differentiated territorial scopes, being more significant in the urban periphery. Among the neighborhoods in these areas, Las Heras and Parque Palermo stand out, framed in the Neighborhood Improvement Program (ProMeBa) financed by the Inter-American Development Bank.

This study analyzes relevant aspects of socio-environmental vulnerability and community resilience in the context of the pandemic in order to lay the foundations for generating urban 
VULNERABILIDAD SOCIOAMBIENTAL Y RESILIENCIA EN DOS BARRIOS DEL BORDE URBANO DE LA CIUDAD DE MAR DEL PLATA: APORTES PARA LA GESTIÓN LOCAL

mia a fin de aportar bases para generar propuestas de intervención urbana que den respuesta a las problemáticas emergentes en el contexto de la pandemia. El procedimiento metodológico empleado se compone de dos enfoques principales: cuantitativo, a partir del cual se construye un Índice de Vulnerabilidad Socioambiental (IVSA) y cualitativo, centrado en entrevistas semiestructuradas a referentes de la comunidad de ambos barrios para abordar aspectos de la resiliencia.

Las condiciones más críticas del IVSA se presentan en un sector de Parque Palermo. La pandemia, además de visibilizar problemas estructurales, ha permitido evidenciar la capacidad de respuesta y de adaptación ante la crisis sanitaria. Sin embargo, estas adaptaciones no se reflejan a nivel urbano. En función de ello, surge el interés de referir al concepto de resiliencia urbana como un eje central de una gestión local sostenible en el escenario de la pospandemia.

\section{Palabras clave: pandemia, resiliencia ba- rrial, periferia urbana, sostenibilidad.}

\section{RESUMO}

A pandemia COVID-19 e as medidas preventivas e obrigatórias de isolamento e distanciamento social transformaram as atividades básicas de produção e reprodução da população. Esses eventos impactaram a dinâmica social e econômica das cidades, gerando e aprofundando as desigualdades. Mar del Plata, Argentina, foi uma das cidades mais afetadas da província de Buenos Aires. Os efeitos tiveram diferentes âmbitos territoriais, sendo mais significativos na periferia urbana. Entre os bairros dessas áreas, destacam-se Las Heras e o Parque Palermo, que integram o Programa de Melhoria de Bairros (ProMeBa), financiado pelo Banco Interamericano de Desenvolvimento. intervention proposals that respond to emerging problems in the context of the pandemic. The methodological procedure used is made up of two main approaches: the quantitative one, from which a Socio-environmental Vulnerability Index (SEVI) is constructed, and the qualitative one, focused on semi-structured interviews with community referents of the community of both neighborhoods for aspects that address resilience.

The most critical conditions of the SEVI occur in a sector of Parque Palermo. The pandemic, in addition to highlighting structural problems, has made it possible to demonstrate the capacity to respond and adaptation to the health crisis. However, these adaptations are not reflected at the urban level. From this, the interest arises to refer to the concept of urban resilience as the central axis of sustainable local management in the post-pandemic scenario.

Key words: pandemic, neighborhood resilience, urban periphery, sustainability.

\section{lience, urban periphery, sustainability.}

Este estudo analisa aspectos relevantes da vulnerabilidade socioambiental e resiliência da comunidade no contexto da pandemia, a fim de lançar as bases para a geração de propostas de intervenção urbana que respondam aos problemas emergentes no contexto da pandemia. O procedimento metodológico utilizado consiste em duas abordagens principais: a quantitativa, a partir da qual é construído o Índice de Vulnerabilidade Socioambiental (IVSA), e a qualitativa, baseada em entrevistas semiestruturadas com referentes da comunidade de ambos os bairros para abordar aspectos de resiliência.

As condições mais críticas do IVSA ocorrem em um setor do Parque Palermo. A 
pandemia, além de tornar visíveis problemas estruturais, permitiu demonstrar a capacidade de resposta e adaptação à crise de saúde. No entanto, essas adaptações não se refletem no nível urbano.
É aí que surge o interesse em se referir ao conceito de resiliência urbana como eixo central da gestão local sustentável no cenário pós-pandêmico.

Palavras-chave: pandemia, resiliência de bairro, periferia urbana, sustentabilidade.

\section{INTRODUCCIÓN}

La enfermedad denominada COVID-19, a causa del virus SARS-CoV-2 despertó en Wuhan, China, a fines de 2019, provocando una transmisión entre personas, en principio por contacto físico directo, pero también a través de objetos compartidos. Sobre la base de un inédito y veloz desarrollo de los contagios que se extendieron por los cinco continentes, la Organización Mundial de la Salud reconoció el evento como pandemia el 11 de marzo de 2020.

Ante la falta de conocimiento profundo, la sorpresa provocada y cierta evidencia de que el contagio se produce en forma directa -persona a persona-, cada país abordó una estrategia distinta, que incluso se diversificó hacia el interior, estableciendo zonas con variadas estrategias y políticas, todas fundadas en gradaciones de aislamiento y distanciamiento social, con la consecuente disminución y paralización de las actividades urbanas.

Estas disposiciones estatales implementadas con la intención de salvaguardar la vida de las personas a través de la combinación de las políticas mencionadas han causado, como contrapartida, reducciones significativas en el aparato productivo, la circulación de mercadería y, por supuesto, el consumo. En la esfera privada -en el ámbito formal-, la consecuencia se refleja en el alto costo económico y social, que tuvo sus efectos principalmente en el "desplome" de los mercados, el cierre de empresas y, como correlato, el despido de gran cantidad de trabajadores.

Las medidas establecidas impactaron de modo distinto según la órbita laboral. Así, el ámbito público y el privado han registrado situaciones contrapuestas en cuanto a los efectos sobre los trabajadores. En Argentina, las estadísticas oficiales arrojan una variación negativa interanual (enero 2020-2021) de -2,9\% en los trabajadores registrados del sector privado, en tanto que en el sector público se percibe un incremento del 0,8\% en el mencionado período. En lo que refiere al personal de casas particulares, el descenso fue aún más significativo, llegando a un $-5,5 \%$. Teniendo en cuenta que las actividades vinculadas a la hotelería, bares, restaurantes, junto al sector de la construcción y servicios representan una fuente importante de ingresos para los hogares más vulnerables, y que éstas redujeron drásticamente sus operaciones durante el período de emergencia, una importante cantidad de personas vio discontinuado su empleo en estas circunstancias.

La situación sanitaria en Argentina instaló un escenario inédito en la primera mitad de 2020 que derivó en el ascenso en los niveles de pobreza (Salvia et al, 2021). Por un lado, el au- 
VULNERABILIDAD SOCIOAMBIENTAL Y RESILIENCIA EN DOS BARRIOS DEL BORDE URBANO DE LA CIUDAD DE MAR DEL PLATA: APORTES PARA LA GESTIÓN LOCAL

mento de los precios de la canasta de consumo continuó avanzando y, por el otro, en el marco de restricciones a la movilidad -ordenadas para contener la subida de contagios-, se limitaron y/o afectaron tanto procesos de trabajo como pautas de consumo (Salvia et al, 2021). Estos hechos han tenido un fuerte impacto en las ciudades, generando y profundizando desigualdades.

La ciudad de Mar del Plata, partido de General Pueyrredon -659.462 habitantes, según estimaciones realizadas a 2021 (INDEC, 2015)- fue uno de los asentamientos con mayores impactos en la provincia de Buenos Aires. La ciudad se ubica al sudeste de la Provincia y posee un marcado perfil turístico que la constituye como uno de los principales centros de veraneo en Argentina. Además de las actividades turísticas, las pesqueras, el sector textil, hortícola alimentario, minero e industrial completan el perfil productivo. Es una ciudad que se ha expandido de manera espontánea y desordenada, generando un área periurbana o zona de interfaz urbano-rural compleja y dispersa. Numerosos sectores que conforman esa zona de interfaz pueden considerarse "áreas de borde", con fuerte dinámica, que manifiestan problemas sociales y ambientales (Zulaica y Ferraro, 2010).

De acuerdo con el informe publicado por el Centro de Estudios Sociales y Políticos (CESP, 2020), los datos de la Encuesta Permanente de Hogares (EPH) correspondientes al segundo semestre de 2019, muestran para el aglomerado Mar del Plata-Batán un 4,3\% de desocupados según la estructura de clases de ocupación. Este valor es mayor al obtenido para el conjunto de aglomerados de Argentina sobre el que se releva la EPH, el cual alcanzó para el período un $3,3 \%$. Por otra parte, la información referida a pobreza e indigencia para el segundo semestre de 2019 indica un total de 18,4\% de hogares bajo la línea de pobreza y un $4,2 \%$ de indigencia en el aglomerado Mar del Plata-Batán. Estos valores, son algo inferiores a los obtenidos por el conjunto de aglomerados relevados en la EPH. En ese sentido, los hogares bajo la línea de pobreza e indigencia alcanzaron $25,9 \%$ y $5,7 \%$, respectivamente.

En este contexto, el desencadenamiento de la pandemia y las medidas de Aislamiento y Distanciamiento Social Preventivo y Obligatorio (ASPO y DISPO) transformaron las actividades básicas de producción y reproducción de la población. Sin duda, los impactos poseen alcances territoriales diferenciados, siendo la capacidad de respuesta diferente en los distintos barrios de Mar del Plata. Los impactos más significativos han tenido lugar en los barrios de la periferia urbana. Entre ellos, se destacan Las Heras y Parque Palermo, barrios localizados al oeste de la ciudad enmarcados en el Programa de Mejoramiento de Barrios (ProMeBa) del Banco Interamericano de Desarrollo ${ }^{1}$. La Figura 1 muestra la localización de la ciudad de Mar del Plata y de los barrios mencionados.

1 El ProMeBa tiene como finalidad mejorar la calidad de vida y contribuir a la inclusión urbana y social e integración de los hogares argentinos de los segmentos más pobres de la población. Su propósito es mejorar de manera sustentable el hábitat de la población que reside en villas y asentamientos irregulares. Mediante la ejecución de proyectos integrales barriales, tiene como objetivos consolidar a la población destinataria en el lugar que habitan, brindando acceso a la propiedad de la tierra, contribuyendo en la provisión de obras de infraestructura urbana, equipamiento comunitario y saneamiento ambiental, y promoviendo el fortalecimiento de su capital humano y social. El Programa es ejecutado por el Ministerio de Desarrollo Territorial y Hábitat, y opera descentralizadamente a través de Unidades Ejecutoras Provinciales (UEP) y Unidades Ejecutoras Municipales (UEM). 

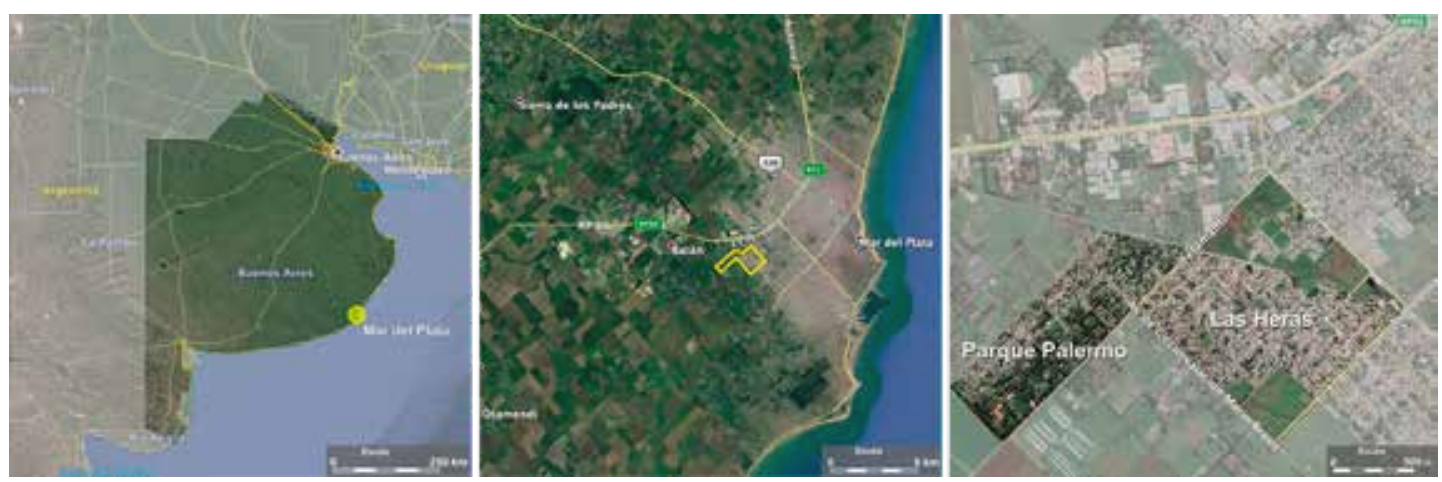

Fig. 1. Localización: Barrios Las Heras y Parque Palermo, Mar del Plata, Partido de General Pueyrredon, Provincia de Buenos Aires, Argentina. Fuente: Elaboración de los autores sobre la base de Google Earth.

Estudios antecedentes (CESP, 2020; INHUS, 2020) demuestran que la actual pandemia por COVID-19 posee alcances territoriales diferenciales en los barrios marplatenses, cuyo potencial de riesgo adquiere implicancias que deben abordarse atendiendo la complejidad y multidimensión de los factores que intervienen en el proceso. De esta manera, el análisis del riesgo brinda elementos clave para su gestión, la cual implica una aproximación procesual que anticipa lo que puede suceder, poniendo el foco en la prevención (Natenzon y Besalú Parkinson, 2020).

Si bien la discusión conceptual del riesgo es amplia (Wynne, 1992; Wilches Chaux, 1993; Beck, 1998), desde el punto de vista analítico, se pueden identificar en principio dos dimensiones constitutivas: la peligrosidad, definida como la probabilidad de ocurrencia de un fenómeno natural o tecnológico con potencialidad de generar daño (Natenzon, 1998); y la vulnerabilidad, que refiere a las condiciones sociales, económicas, culturales, etc, que exponen a una población y la hacen propensa a ser afectada y sufrir daño respecto a una peligrosidad (Lavell, 1996). En este caso en particular, la peligrosidad podría vincularse con la amenaza del COVID-19, en tanto que la vulnerabilidad integra características determinadas por factores o procesos físicos, sociales, económicos y ambientales que aumentan la susceptibilidad de un individuo, una comunidad, activos o sistemas a los impactos de los peligros (UNISDR, 2017). En este sentido, puede definirse como vulnerabilidad socioambiental.

Por otro lado, el concepto de vulnerabilidad se vincula con el de resiliencia, entendido como la capacidad para enfrentar los peligros. A diferencia del concepto de vulnerabilidad, la resiliencia generalmente se considera un atributo positivo y deseable para enfrentar eventos peligrosos (Scherzer et al, 2019).

En el contexto planteado, vale preguntarse ¿la capacidad de respuesta al COVID-19 y a las medidas de ASPO y DISPO es diferente al interior de los barrios Las Heras y Parque Palermo? ¿cómo ha respondido la comunidad de estos barrios a la pandemia? ¿cómo enfrenta la comunidad de los barrios las consecuencias sociales de la pandemia? Los conceptos de vulnerabilidad socioambiental y de resiliencia comunitaria parecieran responder al menos en parte a estos interrogantes.

En términos generales, la vulnerabilidad puede definirse como un proceso multidimen- 
VULNERABILIDAD SOCIOAMBIENTAL Y RESILIENCIA EN DOS BARRIOS DEL BORDE URBANO DE LA CIUDAD DE MAR DEL PLATA: APORTES PARA LA GESTIÓN LOCAL

sional que confluye en el riesgo de la población a sufrir daños ante los cambios o la permanencia de condiciones negativas internas o externas. El nivel de vulnerabilidad, es decir, la capacidad que posee la comunidad para prevenir, reducir o afrontar los riesgos, depende de la interacción de varios factores de distinto origen.

Aunque el concepto de resiliencia se utiliza más frecuentemente para referir al riesgo de desastres, se introduce cada vez con mayor fuerza para analizar la capacidad de respuesta y adaptación ante amenazas, proponiendo una relectura de la vulnerabilidad en términos proactivos. La resiliencia es un concepto complejo con numerosas acepciones (Hallegatte y Engle 2019). Según Asadzadeh et al. (2017), aunque el término resiliencia fue formulado por primera vez en el campo de la ecología por Holling (1973), se ha utilizado desde el siglo XVI y abarca diversos marcos teórico-conceptuales: desde los sistemas socioecológicos hasta desarrollos posteriores en el campo de la sostenibilidad, la mitigación y la adaptación y, más recientemente, la reducción del riesgo de desastres. Siguiendo a los autores, la resiliencia constituye actualmente un tema prioritario en los círculos académicos y en las agendas internacionales $y$ adquiere una relevancia significativa en un contexto de cambio ambiental y en las políticas de planificación urbana. De acuerdo con Ching (2016), resiliencia es un término normativamente atractivo, pero controvertido ya que posee numerosas posibilidades interpretativas. Sin embargo, existe un consenso cada vez mayor en considerar a la resiliencia como la capacidad para adaptarse continuamente a las fluctuaciones sistémicas.

En este marco y considerando el ProMeBa como ámbito de trabajo interdisciplinar de los autores, el presente estudio propone analizar aspectos relevantes de la vulnerabilidad socioambiental en los barrios Las Heras y Parque Palermo y de la resiliencia comunitaria en el contexto de pandemia, a fin de aportar bases para generar propuestas de intervención urbana que den respuesta a las problemáticas emergentes.

\section{MATERIALES Y MÉTODOS}

En función de los objetivos perseguidos, el procedimiento metodológico empleado se compone de dos enfoques principales: cuantitativo y cualitativo. El primero de ellos, se relaciona con el análisis de aspectos relativos a la vulnerabilidad socioambiental en los barrios analizados, en tanto que el segundo aborda la resiliencia comunitaria desde las relaciones propias de una perspectiva interdisciplinar.

El análisis de la vulnerabilidad socioambiental se centró en la construcción de un índice sintético, Índice de Vulnerabilidad Socioambiental (IVSA) que permitió sintetizar distintos aspectos que inciden en la vulnerabilidad. En la construcción del IVSA se consideraron especialmente los aportes de Wilches Chaux (1993) debido a que integra en su enfoque diferentes dimensiones inherentes a la vulnerabilidad. Asimismo, se partió de estudios antecedentes en los cuales se construyeron índices de vulnerabilidad (Zulaica y Ferraro, 2010; Cabral y Zulaica, 2015; Daga et al, 2015), ajustando los indicadores al objetivo de este trabajo. En este caso, la selección de indicadores corresponde a datos censales de 2010 (INDEC, 2010) ${ }^{2} \mathrm{y}$, por lo

2 Aunque los datos oficiales con los que se cuenta de manera desagregada no son actuales, permiten detectar 
tanto, las unidades de referencia espacial fueron los radios censales de ese año. Los datos se procesaron con REDATAM + SP.

Se seleccionaron 17 indicadores en total, algunos de los cuales fueron agrupados en indicadores compuestos. Como se trata de un índice de vulnerabilidad, el mismo expresa las condiciones más críticas de cada indicador; esta es la razón por la que se utilizan indicadores de "costo", que expresan situaciones negativas en el contexto analizado. Luego, los valores obtenidos para cada uno de los indicadores seleccionados se estandarizaron con la finalidad de transformarlos en unidades adimensionales que permitan establecer comparaciones (Buzai, 2003; Buzai y Baxendale, 2006). En este caso, se utilizó la técnica de Puntaje Z . Este procedimiento obtiene el puntaje estándar calculando la sumatoria, el promedio y el desvío estándar de cada indicador. Para el cálculo se utilizó la siguiente fórmula:

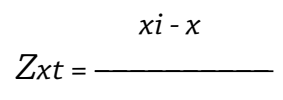

$\sigma$

Donde: $Z x t$ es el puntaje $\mathrm{z}$ de la variable $\mathrm{x}$ para la unidad espacial $i$; $x i$ es la variable para estandarizar; $x$ es la media de la variable x para toda el área de estudio; $\sigma$ es el desvío estándar de la variable x para toda el área de estudio.

En el tratamiento empleado, cada una de las variables es transformada en unidades de desviación típica, siendo las mismas adimensionales, por lo que serán independientes de las unidades en las que se expresaban originalmente. De esa manera es posible comparar

comparativamente las diferencias más significativas al interior de los barrios. entre sí variables referentes a tópicos distintos (García de León, 1997).

Posteriormente, se aplicó la técnica del linkage analysis (Buzai, 2003) para construir una matriz de correlaciones que permitió detectar fuertes asociaciones entre los indicadores y distinguir aquellos que podrían ser redundantes (Giraud-Herrera y Morantes-Quintana, 2017). De esta manera se identificaron las máximas correlaciones y se obtuvieron los "pares recíprocos" en cada caso, que definen indicadores compuestos. Un indicador compuesto es una representación simplificada que busca resumir un concepto multidimensional en un índice simple (unidimensional) con base en un modelo conceptual subyacente (Schuschny y Soto, 2009). En relación a los "pares recíprocos", se tomó 0,5 como límite mínimo de valor de correlación significativa.

En la Tabla 1 se presentan los indicadores e indicadores compuestos considerados en la construcción del IVSA, el cual se calculó a partir de la sumatoria de los valores estandarizados de cada indicador o indicador compuesto. Los resultados obtenidos para el índice se representaron espacialmente utilizando un Sistema de Información Geográfica. Se utilizó para ello QGIS (versión 3.10) y se analizaron los aspectos más relevantes de la configuración espacial a partir de la clasificación de los resultados obtenidos por desvíos estándar. Se definieron 4 categorías de vulnerabilidad: muy superior a la media del conjunto, superior a la media, inferior a la media y muy inferior a la media.

Para el análisis de aspectos clave en la resiliencia comunitaria se utilizó un enfoque cualitativo, ya que permite lograr un mayor conocimiento y comprensión de las percepciones, opiniones y puntos de vista de referentes 
VULNERABILIDAD SOCIOAMBIENTAL Y RESILIENCIA EN DOS BARRIOS DEL BORDE URBANO DE LA CIUDAD DE MAR DEL PLATA: APORTES PARA LA GESTIÓN LOCAL

TABLA 1. INDICADORES E INDICADORES COMPUESTOS UTILIZADOS EN LA CONSTRUCCIÓN DEL ÍNDICE DE VULNERABILIDAD SOCIOAMBIENTAL

\section{Indicadores - indicadores compuestos}

Porcentaje de población con Necesidades Básicas Insatisfechas

Porcentaje de población desocupada

Porcentaje de población de 0 a 14 años de edad - Porcentaje de población que no sabe leer ni escribir

Porcentaje de población de más de 65 años

Porcentaje de hogares en viviendas con régimen de ocupación

Porcentaje de hogares sin computadora

Porcentaje de hogares sin celular

Porcentaje de hogares sin baño de uso exclusivo

Porcentaje de hogares con agua fuera de la vivienda - Porcentaje de hogares con calidad constructiva insuficiente

Porcentaje de hogares que no utilizan agua de red para beber o cocinar

Porcentaje de hogares sin cobertura de red cloacal - Porcentaje de hogares con calidad de conexión a los servicios básicos insuficiente

Porcentaje de hogares sin gas de red

Porcentaje de hogares sin heladera

Porcentaje de hogares con Calidad de materiales de la vivienda IV

Fuente: Datos obtenidos del INDEC (2010).

de la comunidad. La elección de este enfoque se fundamenta en que el análisis de la resiliencia se centra en indagar acerca de los significados atribuidos a los hechos por parte de los referentes de ambos barrios, considerando fenómenos dentro del propio entorno en el cual ocurren. De acuerdo con Baeza (2002), la investigación cualitativa permite un acercamiento a la realidad social al destacar la mirada subjetiva de los sujetos, la complejidad de los fenómenos, su historicidad y la imagen sistémica que estos sujetos atribuyen a los hechos.

Partiendo de lo anterior, se realizaron entrevistas semiestructuradas a referentes clave $^{3}$ de ambos barrios que desarrollan sus

3 Las entrevistas se realizaron en el marco del actividades en instituciones, organizaciones, sociedades de fomento, comedores comunitarios. Se llevaron a cabo once entrevistas presenciales, considerando seis referentes de Las Heras y cinco de Parque Palermo. Para ello, se definieron en conjunto con los entrevistados los lugares de encuentro, respetando las medidas de distanciamiento. Las entrevistas, realizadas durante los meses de marzo y abril de 2021, fueron organizadas siguiendo un guión que se desarrolló a partir de preguntas abiertas. En la entrevista semi-estructurada, el trabajo investigativo se organiza a partir de ejes temáticos de reflexión y/o a partir de preguntas orienta-

Diagnóstico Integral 2021 llevado a cabo en el marco del ProMeBa. 
doras; así, la lista de ejes y/o preguntas, permite verificar que haya sido recabada la misma información de cada uno de los sujetos entrevistados (Tonon, 2015). La decisión de utilizar este tipo de entrevistas radica en poder desarrollar un diseño flexible en el cual los referentes ocupan un lugar protagónico, aportando al estudio de la resiliencia comunitaria.

A los fines del presente trabajo, los ejes principales sobre los que se organizó el guión fueron los siguientes: 1) información general sobre el entrevistado, incluyendo su trayectoria y actividades realizadas en el barrio; 2) características generales de los barrios relativas a población, actividades, infraestructura, servicios, presencia de instituciones (antes y durante la pandemia); 3) problemas y debilidades observados en el barrio, identificando aquellos que poseen características estructurales por un lado $y$, por el otro, los que se han generado o intensificado en el contexto de pandemia: 4) fortalezas que poseen los barrios para dar respuesta a problemas estructurales y fortalezas que devienen de la capacidad de respuesta frente a los problemas derivados de la pandemia; y 5) proyectos, propuestas, actividades proyectadas en 2021.

La realización de entrevistas fue complementada con estrategias de observación y trabajo de campo que permitieron corroborar la información aportada por los entrevistados. El análisis de los datos demandó la estrategia metodológica denominada análisis temático. Esta estrategia facilita la identificación y análisis de los datos para determinar los temas y estructuras involucrados en el concepto de resiliencia a través de las experiencias, significados y realidades de los sujetos. Esto permite sistematizar los resultados y analizarlos desde un enfoque inductivo.

\section{MAR DEL PLATA Y LOS BARRIOS LAS HERAS Y PARQUE PALERMO}

El origen de la ciudad de Mar del Plata se remonta al año 1874 y reconoce, como uno de los hechos salientes y singulares, el haberse apartado en su acto fundacional de los preceptos que a tal fin establecía la Ley General de Ejidos de 1870 (que regía para el ámbito de la provincia de Buenos Aires), dado que se erigió sobre un loteo de tierras privadas y no sobre tierras fiscales, tal como lo preveía el mencionado marco regulatorio. Dicha particularidad, se ha encontrado emparentada con la preeminencia que ha tenido el capital inmobiliario en la evolución de la subdivisión, uso y ocupación del suelo en el devenir histórico de la ciudad.

Otra situación característica es la coexistencia en su ejido urbano (delimitación del área urbana principal) de dos trazas o rumbos disímiles. Por un lado, el damero original, que se corresponde con la fundación de la ciudad, se extiende entre el Mar Argentino, el Arroyo La Tapera, predios del área rural en el extremo noroeste y la avenida Juan Bautista Justo; y por otro, a partir de la mencionada avenida y hacia el sur, el rumbo que presenta el resto del Partido y la totalidad de la provincia de Buenos Aires.

La extensión de la ciudad se ha dado históricamente a partir de reiterados impulsos del capital privado a través de operaciones de transformación de suelo rural en suelo urbano, en muchos de los casos por iniciativas con débiles condicionantes por parte del Estado. Desde finales del Siglo XX se ha ido consolidando un proceso selectivo de valorización del territorio, a partir de impulsos del capital inmobiliario, orientados a la demanda de los sectores más solventes de la población y facilitados por la 
VULNERABILIDAD SOCIOAMBIENTAL Y RESILIENCIA EN DOS BARRIOS DEL BORDE URBANO DE LA CIUDAD DE MAR DEL PLATA: APORTES PARA LA GESTIÓN LOCAL

flexibilidad de las normas urbanísticas (Mignaqui, 2012) que, como contrapartida, impacta negativamente en los sectores más vulnerables.

En los barrios Las Heras y Parque Palermo, se plantea uno de los casos a los que Pírez (2016) denomina de "urbanización inversa", recurrentes en América Latina. El capital desarrollador se limitó a la generación de una débil trama de calles, formando manzanas de diversos tamaños, sin acompañar ningún otro tipo de infraestructura o acondicionamiento que permitiera dar soporte de las construcciones $\mathrm{y}$, consecuentemente, a la futura ocupación del espacio. La indiferencia de la trama respecto de las condiciones de la topografía se pone de manifiesto al transitar los barrios, sobre todo en época de lluvias: allí se perciben, no solamente aquellas zonas más deprimidas del territorio sino, también la ausencia de infraestructura que subsane esa condición.

Se trata de un área que podría interpretarse en términos de "borde urbano". Según Garay (2000), definir un borde como límite del perímetro urbanizado implica afirmar que es un ámbito de relación entre lo rural y lo urbano. La noción de ámbito la distingue del concepto de línea, para comenzar a referirse a una superficie, a una banda territorial donde lo urbano y lo rural, en cierto modo, conviven. Es en esta zona donde la influencia de las características propias de lo urbano va dejando paso a las cualidades propias de la zona rural, situación que también sucede en el sentido contrario.

En función de lo anterior, el área en la que se localizan los barrios Las Heras y Parque Palermo se caracteriza por presentar una ocupación débil y fragmentaria del suelo: parcelas de grandes tamaños con formas irregulares, porosidad edilicia con bajas densidades edifi- catorias y escasas calles, cuando éstas no son inexistentes. Las cualidades espaciales y funcionales de los territorios fronterizos -sobre todo aquellos obstáculos de dimensiones suficientemente grandes para desagregar un espacio-, una vez eliminados o sustituidos, generan terrenos con una dimensión que posibilita el proyecto de transformación de gran escala.

Este espacio se identifica por su elevada entropía ${ }^{4}$ urbanística, y su falta de estructuración con el resto. Además, en él suelen coexistir usos marginales diversos que, por razones económicas o de compatibilidad, no pueden emplazarse dentro del núcleo urbano (actividades comerciales, industriales o agrícolas, en ocasiones incompatibles con usos residenciales). A pesar de contar con una morfología imprecisa y en constante transformación, se observan en sectores específicos límites precisos, como es el caso de la traza de la vía del ferrocarril. Una característica común que define a estos espacios es la situación de vulnerabilidad socioambiental en que se encuentran y las diferenciaciones internas entre distintos sectores; es decir, la capacidad de respuesta de la población en función de las condiciones sociales, sanitarias y económicas -fundamentalmente- difiere al interior de los barrios.

\section{LA VULNERABILIDAD SOCIOAMBIENTAL}

Wilches Chaux (1993) define la vulnerabilidad como la incapacidad de una comunidad para absorber, mediante el autoajuste, los efectos de un determinado cambio en su entorno, es decir,

4 Entropía entendida aquí, no como degradación gradual de la energía, sino como medida del desorden. 


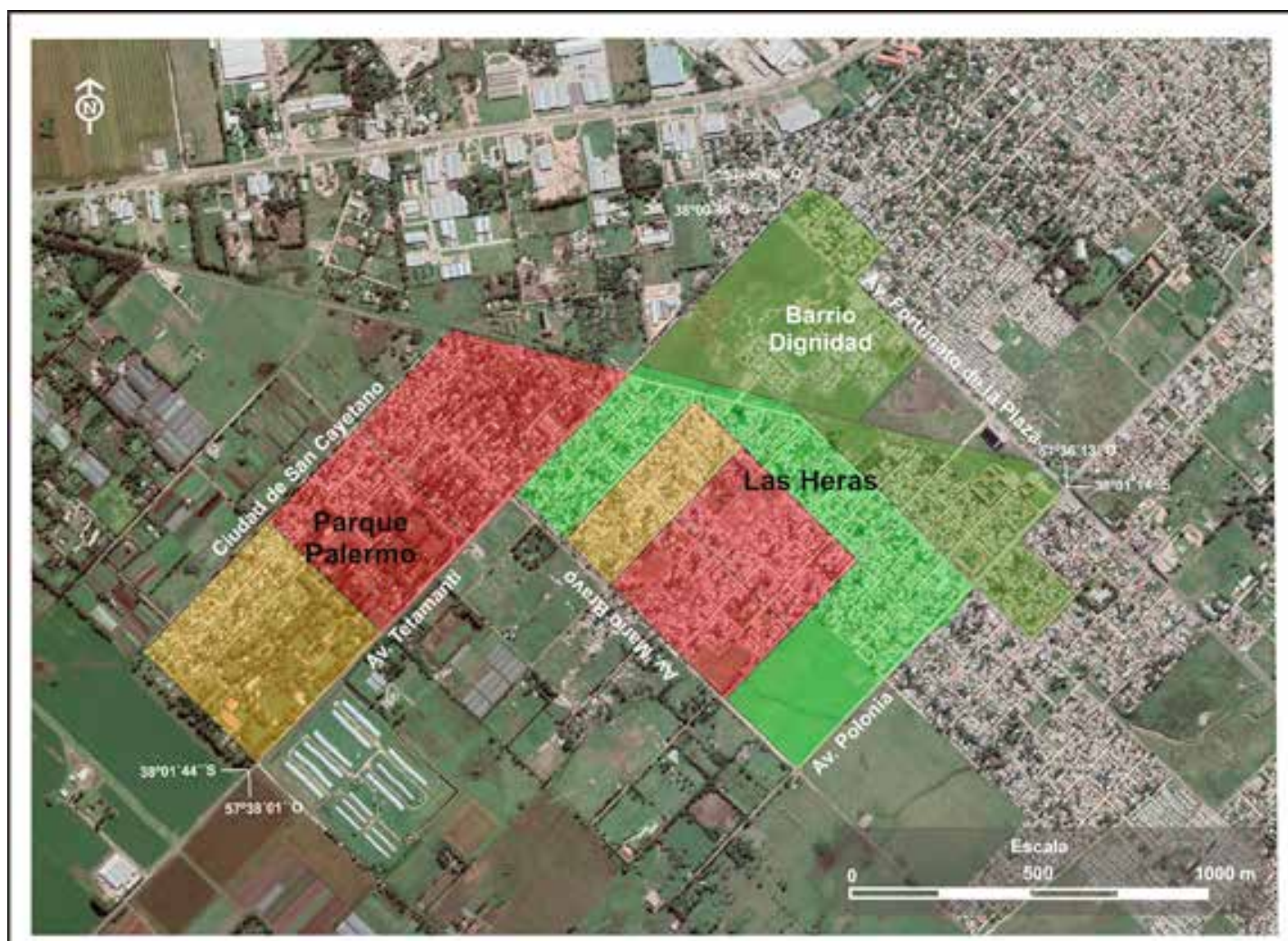

Referencias

Indice de Vulnerabilidad Socioambiental (IVSA)

Fig. 2. Barrios Las Heras y Parque Palermo: Índice de Vulnerabilidad Socioambiental (IVSA). Fuente: Elaboración de los autores sobre la base de Google Earth.

su inflexibilidad o incapacidad para adaptarse a ese cambio. Sánchez González y Egea Jiménez (2011) señalan que hay investigaciones que abordan las interacciones entre las cuestiones ambientales y sociodemográficas abarcando ámbitos diversos, siendo el urbano el espacio más atractivo para este tipo de investigaciones, debido a que en él la vulnerabilidad se asocia con riesgos crecientes y dinámicos.
Dado que los factores que inciden en la vulnerabilidad son internos y también externos (determinados por las condiciones del ambiente) es posible hacer referencia a condiciones de vulnerabilidad socioambiental (Zulaica y Ferraro, 2016). Este tipo de vulnerabilidad, definida en estudios previos (Zulaica y Ferraro, 2010; Cabral y Zulaica, 2015; Daga et al, 2015) está determinada por las características intrínsecas 
VULNERABILIDAD SOCIOAMBIENTAL Y RESILIENCIA EN DOS BARRIOS DEL BORDE URBANO DE LA CIUDAD DE MAR DEL PLATA: APORTES PARA LA GESTIÓN LOCAL

de la población y su entorno próximo para enfrentar las dificultades o riesgos. Dichas características comprenden un conjunto de factores (sanitarios, educativos, habitacionales, económicos, sociales, entre otros) cuyo grado de debilidad define distintos niveles de vulnerabilidad socioambiental para enfrentar los peligros.

El IVSA construido conforma una herramienta para monitorear las tendencias de vulnerabilidad a largo plazo desde un punto de vista retrospectivo. La información que contiene puede contribuir a la elaboración de proyecciones a corto plazo, aportando a la toma de decisiones hacia el futuro. El análisis de la distribución de IVSA (Figura 2) intenta profundizar en las diferenciaciones al interior de los barrios analizados.

Los datos obtenidos para el área de estudio revelan situaciones críticas respecto del resto de la ciudad de Mar del Plata. Asimismo, se evidencian diferencias al interior de los barrios analizados. En ese sentido, las condiciones más desfavorables se presentan en un importante sector de Parque Palermo y, en Las Heras, en el área próxima a la avenida Mario Bravo. En contraposición, las condiciones más favorables se exhiben en Las Heras, en las áreas más cercanas a la avenida Fortunato de la Plaza. En este rango mencionado se encuentran el barrio de viviendas sociales correspondientes al Plan Dignidad 5 .

5 De acuerdo con Zulaica y Rampoldi Aguilar (2009), el Plan Dignidad es un plan de viviendas sociales financiado por la Provincia de Buenos Aires para atender a las demandas habitacionales de los municipios. Se trata de casas de hasta tres dormitorios y dúplex, con cocinas y baños equipados, erigidas sobre lotes de 200 m2. En el Barrio Las Heras se construyeron 200 de las viviendas previstas por el Plan en Mar del Plata. Los destinatarios de las mismas en su totalidad son 430 familias que ocupaban seis manzanas en la zona
El comportamiento general del IVSA guarda estrecha relación con el indicador de $\mathrm{Ne}$ cesidades Básicas Insatisfechas (NBI). Las NBI permiten identificar a la población o a los hogares que manifiestan importantes limitaciones en su vivienda (espacio insuficiente, estructura precaria o falta de instalaciones sanitarias), en la escolaridad de los niños o en la capacidad de generar recursos económicos. El enfoque de las NBI capta a la población considerada como pobres estructurales, es decir aquella que requiere una importante inversión material o esfuerzo personal para superar el estado de precariedad social en la que se encuentran. El análisis de la distribución de la población con NBI en los barrios, muestra situaciones más desfavorables en Parque Palermo, especialmente en el sector próximo a la vía y en Las Heras en un área lindera a la avenida Mario Bravo, coincidiendo con los valores más altos de población desocupada.

Respecto de la educación, la presencia de población que no sabe leer ni escribir revela condiciones más críticas en un sector de Parque Palermo con más del 10,1\% de la población en esa situación. A su vez, en este barrio se presentan los porcentajes más altos de población infantil. Un dato relevante a considerar en el contexto actual es la presencia de personas mayores de 65 años. Los datos del último censo nacional aportan una idea general de esa distribución cuyo análisis permite destacar la localización de dicha población fundamentalmente

de la Av. Juan José Paso y Alsina (Villa de Paso). Este asentamiento data de los años 60, cuando la demanda de mano de obra atrajo a trabajadores del interior (especialmente de Santiago del Estero) que nunca regresaron a sus provincias. El Plan Dignidad no aporta soluciones habitacionales a las familias de los barrios en estudio, sino que las viviendas fueron construidas para atender las necesidades de otras zonas de Mar del Plata. 
en el barrio Las Heras, en el sector próximo a la Avenida Polonia, donde los valores superan el 8,0\%.

El porcentaje de hogares en viviendas con régimen de ocupación ${ }^{6}$ es frecuente en los barrios analizados. Los datos del último censo indican una zona central y próxima a las vías en el barrio Las Heras, donde este régimen caracteriza a más del 13,28\% de los hogares. La regularización de la tenencia de la tierra, conforma un punto de partida central para garantizar el acceso al suelo urbano. Si bien no se dispone de datos cuantitativos actualizados, este fenómeno se ha intensificado en los últimos tiempos y en algunos sectores va de la mano de las ocupaciones más recientes que caracterizan las áreas de expansión.

Desde el punto de vista habitacional, la calidad constructiva de la vivienda es un indicador que tiene en cuenta la calidad de los materiales con los que está construida y las instalaciones internas a servicios básicos (agua de red y desagüe) de las que dispone. En este sentido, se diferencian tres categorías principales: 1) calidad constructiva satisfactoria que refiere a las viviendas que disponen de materiales resistentes, sólidos y con la aislación adecuada; a su vez también disponen de cañerías dentro de la vivienda y de inodoro con descarga de agua; 2) calidad constructiva básica, que no cuentan con elementos adecuados de aislamiento o tienen techo de chapa o fibrocemento pero, al igual que la categoría anterior, poseen cañerías dentro de la vivienda y de inodoro con descarga de agua; y 3) calidad insuficiente, que engloba a las viviendas que no cumplen ninguna de las condiciones anteriores. Cuando se analizan

6 La ocupación puede ser con o sin permiso de los propietarios. los datos correspondientes a los radios censales del área, se observa una distribución desigual de las viviendas con calidad constructiva insuficiente, alcanzando las condiciones más críticas en Parque Palermo, con valores que superan el 31,53\%.

Otro indicador habitacional interesante, es el que tiene que ver con la calidad de los materiales con que están construidas las viviendas (material predominante de los pisos y techos), teniendo en cuenta la solidez, resistencia y capacidad de aislamiento, así como también su terminación. En función de ello, se definen cuatro categorías principales: 1) calidad I, significa que la vivienda presenta materiales resistentes y sólidos tanto en el piso como en techo; presenta cielorraso; 2) calidad II, que indica situaciones en las cuales la vivienda presenta materiales resistentes y sólidos tanto en el piso como en el techo; no posee techo ni cielorraso o bien materiales de menor calidad en pisos; 3 ) calidad III, que incluye viviendas poseen materiales poco resistentes y sólidos en techo y en pisos; y 4) calidad IV, la vivienda presenta materiales de baja calidad en pisos y techos. Los datos procesados para el barrio, permiten destacar mayor concentración de viviendas con calidad IV en Parque Palermo, especialmente en el sector más próximo a las vías. En el barrio Las Heras, las situaciones más desfavorables se presentan en la zona más próxima a la avenida Tetamanti. La Figura 3 muestra las condiciones habitacionales de las familias de ambos barrios.

La calidad de conexión a servicios básicos refiere al tipo de instalaciones con que cuentan las viviendas para su saneamiento. Para este indicador, se utilizan las variables procedencia del agua y tipo de desagüe. En función de ello, se definen tres categorías: 1) calidad satisfacto- 
VULNERABILIDAD SOCIOAMBIENTAL Y RESILIENCIA EN DOS BARRIOS DEL BORDE URBANO DE LA CIUDAD DE MAR DEL PLATA: APORTES PARA LA GESTIÓN LOCAL

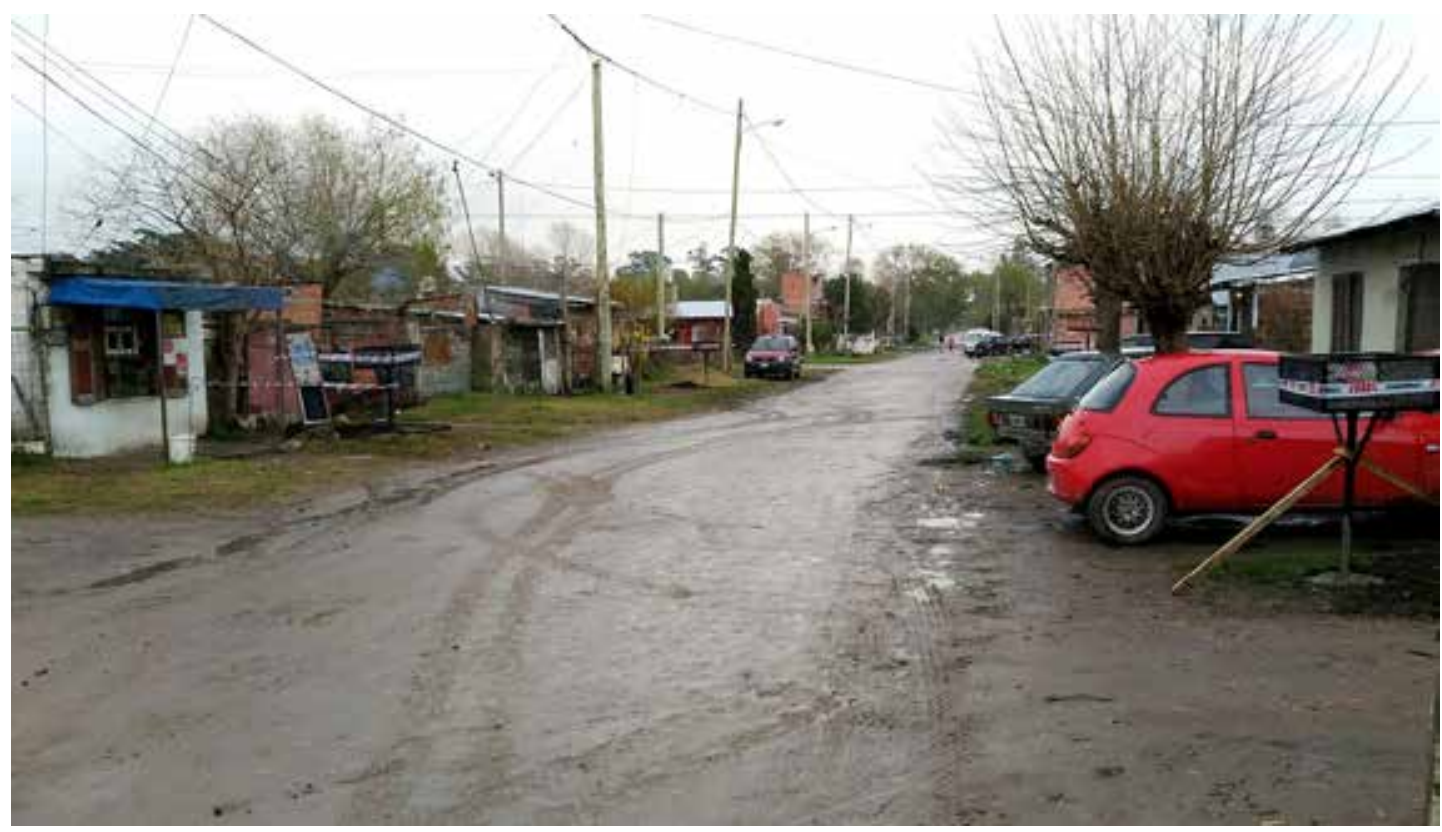

Fig 3 a

Fig $3 b$

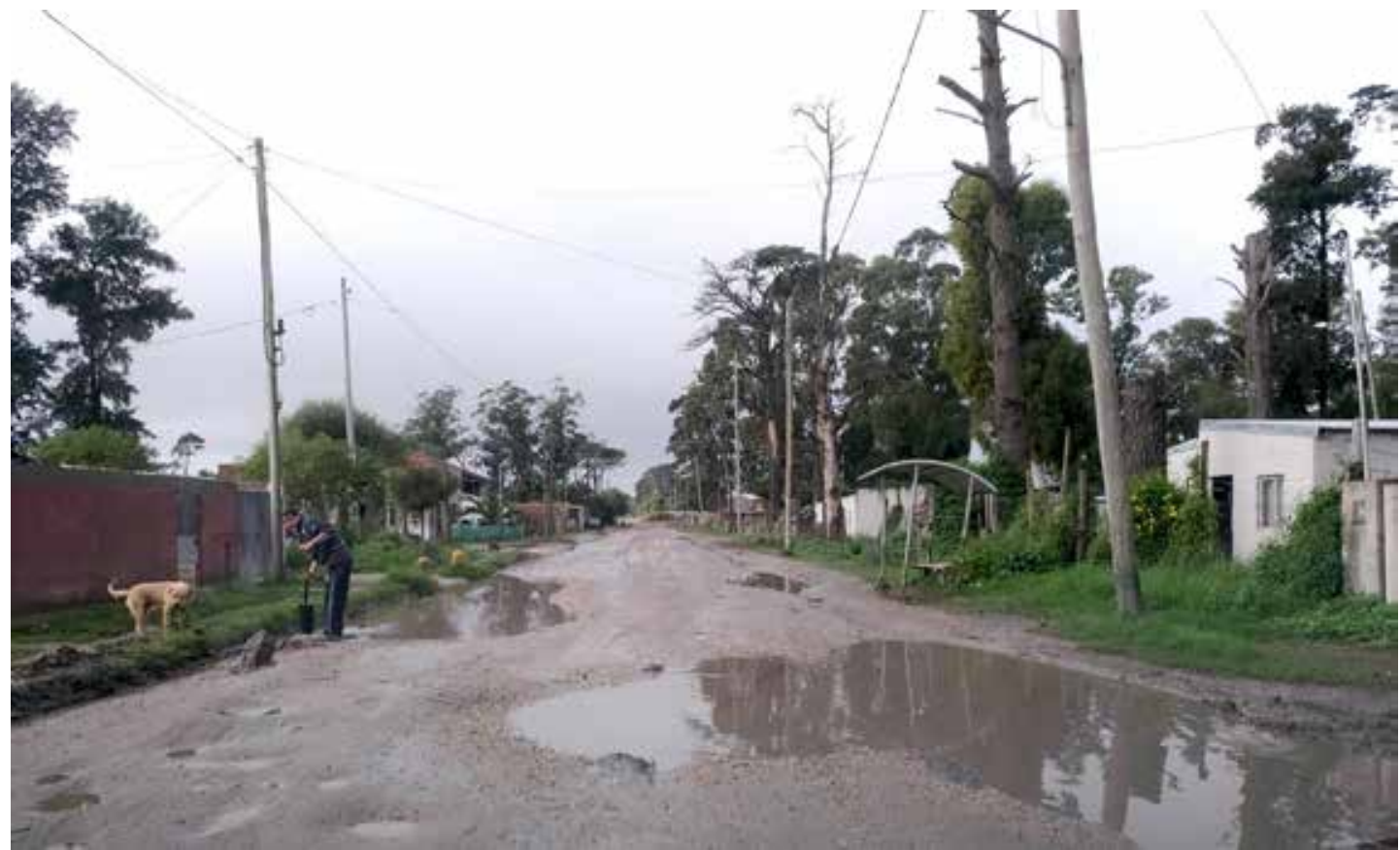




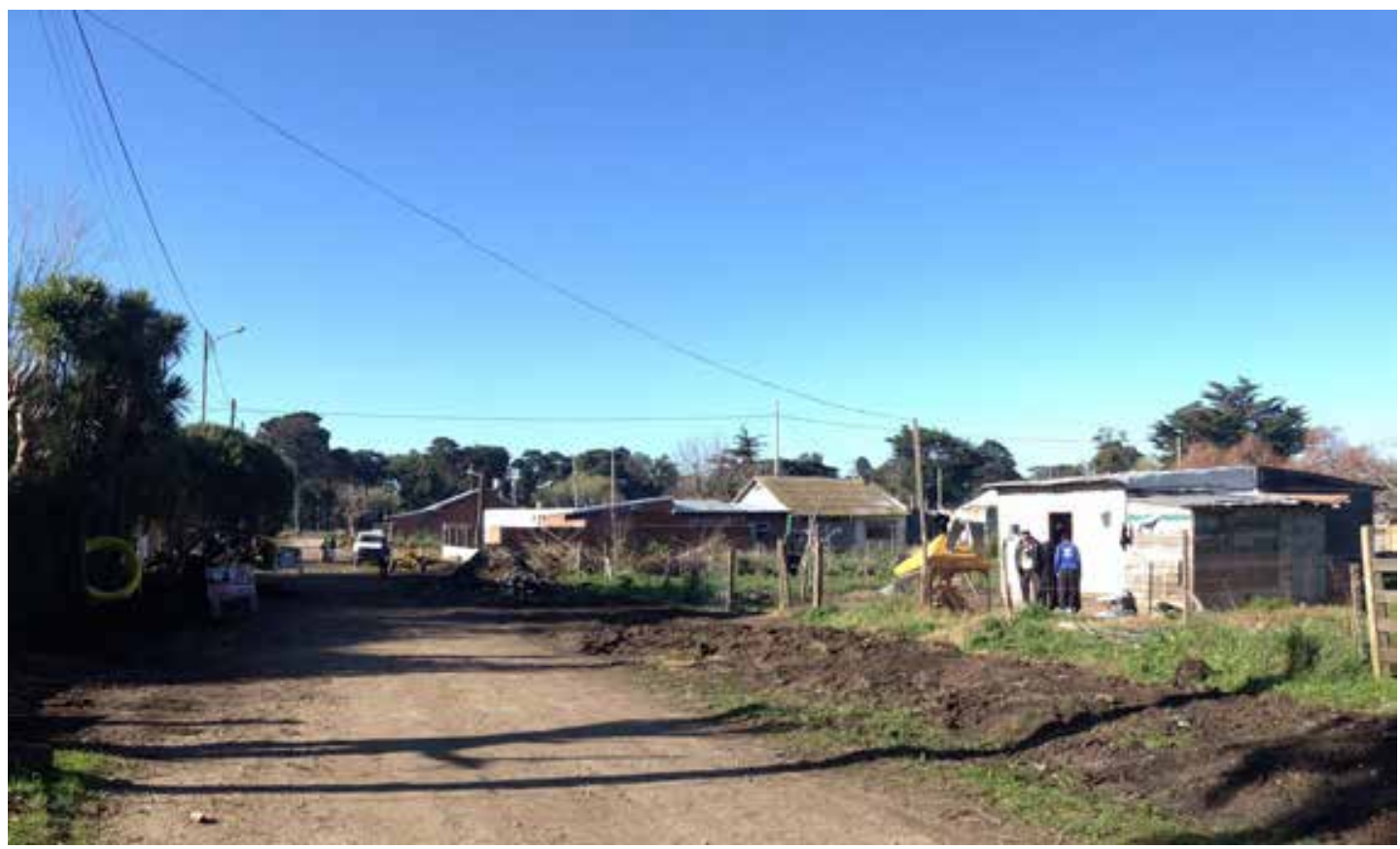

Fig. 3.a,b,c y d. Barrios Las Heras y Parque Palermo: condiciones habitacionales (2021). Fuente: los autores.

Fig $3 d$

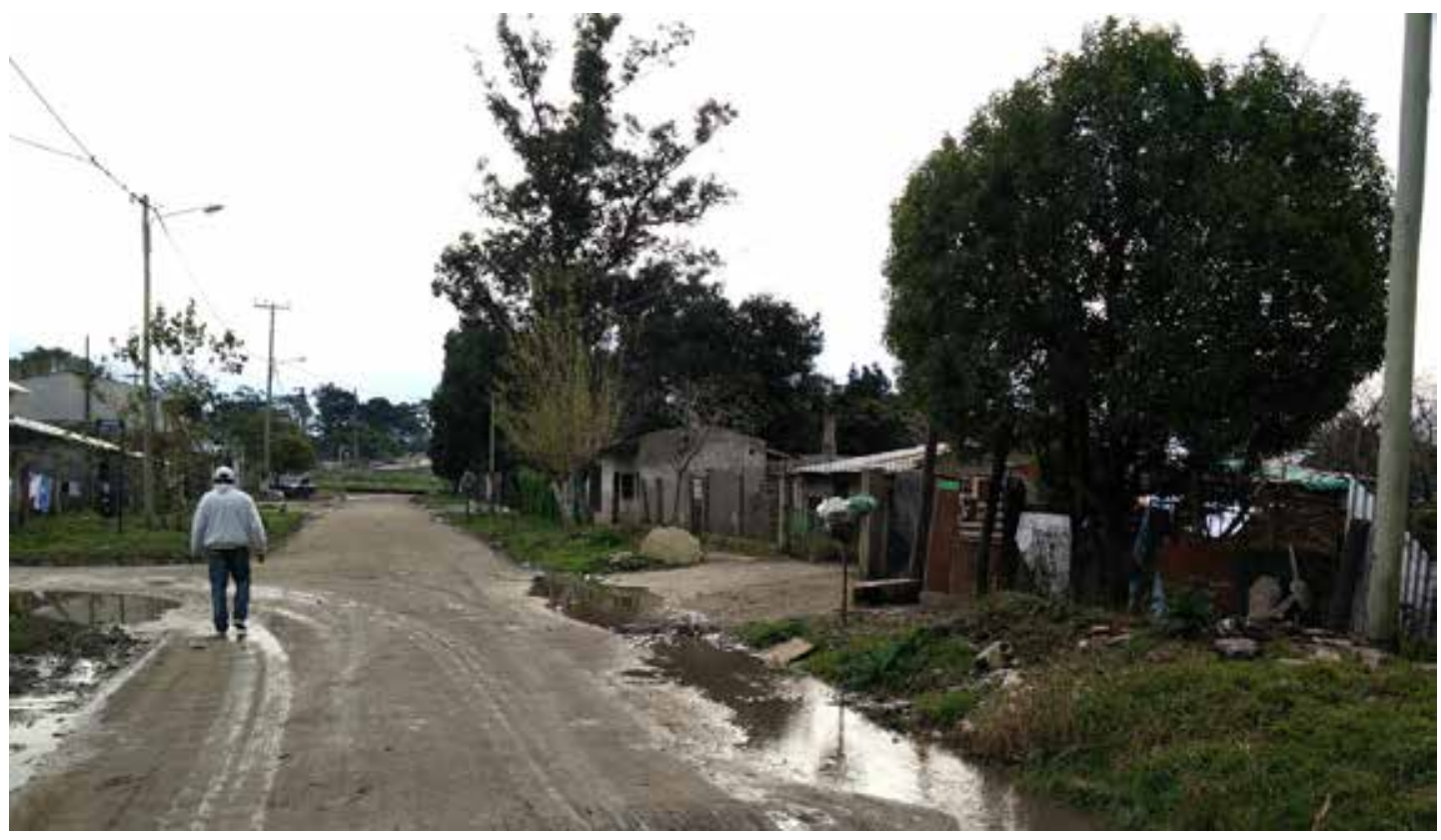


VULNERABILIDAD SOCIOAMBIENTAL Y RESILIENCIA EN DOS BARRIOS DEL BORDE URBANO DE LA CIUDAD DE MAR DEL PLATA: APORTES PARA LA GESTIÓN LOCAL

ria, que refiere a las viviendas que disponen de agua a red pública y desagüe cloacal; 2) calidad básica, que describe la situación de aquellas viviendas que disponen de agua de red pública y el desagüe a pozo con cámara séptica; y 3) calidad insuficiente que agrupa a las viviendas que no cumplen ninguna de las dos condiciones anteriores. El análisis de la distribución de este indicador en los barrios permite señalar que las condiciones más críticas (calidad insuficiente) se presentan en el barrio Parque Palermo con más del 19,4\% de viviendas en esta condición y especialmente en el área más próxima a las vías que supera el $61,0 \%$.

La presencia de baño o letrina de uso exclusivo y la procedencia del agua para beber y cocinar son indicadores centrales en el marco de la crisis sanitaria generada por el COVID-19. El primero de ellos indica si las personas del hogar comparten en forma habitual el baño con miembros de otro hogar. En este sentido, conforma un indicador habitacional útil para conocer las condiciones sanitarias. El análisis de la información censal relativa a los hogares que comparten el baño revela situaciones más desfavorables en un sector de Las Heras próximo a la avenida Mario Bravo con más de 5,56\% de los hogares en esa condición.

Cuando se analiza la procedencia del agua para beber y cocinar, se detecta un sector sobre el barrio Las Heras con más de un 2,9\% de los hogares que no utilizan agua de red. No obstante, los porcentajes más altos de hogares con agua fuera de la vivienda se detectan en Parque Palermo (más del 15,5\%). Por su parte, en relación a la red cloacal, los datos indican que más del 24,6\% de los hogares de Parque $\mathrm{Pa}$ lermo no poseían desagüe a la red pública. Por otro lado, el análisis de los hogares que poseen gas de red para cocinar y calefaccionarse muestra diferencias significativas entre Las Heras y Parque Palermo. En ese sentido, este último barrio presentaba, según los datos censales, más del 76,8\% de los hogares sin acceso al servicio. En cuanto a la disponibilidad de heladera para garantizar la conservación de los alimentos, los valores más altos se presentan en Parque Palermo superando el 10,0\%.

Los datos censales, muestran en numerosos sectores de los barrios, la ausencia de computadora es un hecho frecuente, especialmente en Parque Palermo, en el sector próximo a las vías. La distribución de los hogares que no poseían celular refleja una condición semejante, aunque los valores son inferiores. Este indicador también adquiere importancia en el contexto de pandemia, dado que la "virtualidad" para el desarrollo de numerosas actividades cotidianas -educativas, por ejemplo-demanda disponer de dispositivos electrónicos.

Aunque los datos cuantitativos han sufrido modificaciones en el tiempo, sobre todo ligados a la extensión de la infraestructura y servicios básicos, las diferenciaciones internas continúan e incluso se han intensificado condiciones de vulnerabilidad en sectores específicos.

Más allá de la información aportada a partir de los datos cuantitativos de vulnerabilidad socioambiental interesa indagar, en este caso, acerca de cómo las comunidades de estos barrios han enfrentado la pandemia desde sus propias capacidades y habilidades. Esta respuesta positiva de la población para afrontar la adversidad en el marco de una crisis puede interpretarse desde el enfoque de la resiliencia comunitaria. 


\section{LA RESILIENCIA COMUNITARIA}

De acuerdo con Maguire y Cartwright (2008), la resiliencia comunitaria identifica la manera en que las personas responden a las adversidades que les afectan tales como terremotos, inundaciones, sequías, heladas, atentados, represión política, entre otras, como es la pandemia por COVID-19. Además, muestra cómo se desarrollan y fortalecen los recursos que la comunidad posee.

En este sentido, la resiliencia comunitaria puede definirse como la condición colectiva para hacer frente a las adversidades y sobreponerse (Joerin et al, 2012). Se trata de un proceso dinámico cuyo resultado es la adaptación positiva en contextos de crisis (Wilson, 2013). Esto implica la capacidad de las comunidades para anticiparse y adaptarse a los riesgos y recuperarse sin comprometer sus posibilidades a largo plazo. Así, se incluye la capacidad para desarrollar habilidades, comportamientos y acciones para superar la adversidad.

El conocimiento del área y el análisis de las entrevistas realizadas permite sostener que, en Las Heras y Parque Palermo, las actividades económicas desempeñadas por la población para su sustento se desarrollan en condiciones de informalidad ${ }^{7}$. En particular, en este caso

7 En Argentina, sobre una población total del país estimada en 45,8 millones de habitantes, en enero de 2021 la cantidad de trabajadores registrados asciende a 11,9 millones, representando un $26,4 \%$ de la población. Si se considera un promedio histórico del 39,3\% correspondiente a la Población Económicamente Activa (PEA), una comparación expeditiva permite inferir la existencia de un alto porcentaje de trabajo no registrado, en el orden del $18.6 \%$ del cual no es posible obtener variaciones estadísticas por realizar actividades en informalidad. Es justamente en las áreas urbanas abordadas en el presente estudio que se asienta esta población que se encuentra, en su mayoría, tienen que ver con la construcción, la industria del pescado, en tanto que una proporción significativa se desempeñan como changarines. También se destaca la presencia de personal de casas particulares, en especial de mujeres. Las actividades productivas vinculadas con la horticultura son en su mayoría temporales y se limitan a Parque Palermo. Son comunes en ambos barrios las actividades de cirujeo, donde las familias viven de la separación y recuperación de materiales obtenidos de la basura que luego comercializan. En 2020, el contexto de pandemia ha acentuado esta actividad como consecuencia de la pérdida de fuentes laborales.

Ligado a lo anterior, la información recabada en las entrevistas permite sostener que ha aumentado la cantidad de familias cuya vida y subsistencia está atravesada por la participación en el predio de disposición final de residuos sólidos urbanos de la ciudad Mar del Plata. La dificultad para acceder a fuentes de trabajo es un hecho que se menciona reiteradamente durante las entrevistas. Por otra parte, el trabajo no registrado e informal es una característica frecuente en los barrios, situación que impide gozar de los beneficios de la previsión social.

En Parque Palermo, personal directivo de la escuela del barrio remite a los problemas haciendo referencia a las NBI que caracterizan a la población. Sostiene que los problemas del barrio son estructurales y enfatiza en las críticas condiciones de vivienda, sanitarias, de hacinamiento y en la sinergia con la informalidad de las actividades que desarrollan. Por su parte, una referente de Las Heras menciona que este barrio reúne todas las problemáticas que carac-

en situación de vínculo informal con sus actividades laborales. 
VULNERABILIDAD SOCIOAMBIENTAL Y RESILIENCIA EN DOS BARRIOS DEL BORDE URBANO DE LA CIUDAD DE MAR DEL PLATA: APORTES PARA LA GESTIÓN LOCAL

terizan a los barrios vulnerables y enfatiza en su carácter estructural, destacando que se han acentuado en el contexto actual por la ausencia o disminución de fuentes de trabajo.

La resiliencia comunitaria depende de la capacidad del sistema y de los individuos expuestos a desastres, crisis y vulnerabilidades para anticiparse, planificar, enfrentar, recuperarse y adaptarse a las amenazas (Castro Díaz, 2013; Brooks et al, 2014; Ellis y Abdi, 2017; Visintini y Castro Díaz, 2018). La adaptación de las familias para generar nuevas fuentes de ingreso es parte de esa resiliencia, como lo es también la readecuación de la vida al interior de los hogares para atender las necesidades emergentes.

El aislamiento y distanciamiento social no son condiciones que puedan sostenerse en el tiempo. Así lo indican las familias cuyos hogares son, en general, multifamiliares, extendidos, ensamblados y con numerosos niños, que habitan en condiciones de hacinamiento y deben salir a buscar fuentes de ingresos.

Ligado a lo anterior, la calidad educativa en estos barrios se ha visto afectada en 2020. La inclusión digital es una temática clave, dada la importancia fundamental de las nuevas tecnologías en lo referido a la inserción laboral y social de cada individuo. La problemática de disponibilidad de equipos y de conectividad fueron mencionadas por los referentes de los establecimientos educativos de los barrios en más de una oportunidad. A esto se le suma la falta de acompañamiento familiar que responde, entre otras cuestiones, a la necesidad de salir a trabajar. No obstante esta situación crítica, desde los establecimientos educativos se han generado estrategias de conexión y comunicación a través de redes comunitarias que, como señalan los referentes, han fortalecido los lazos para dar respuesta a las necesidades. En virtud de ello, se ha trabajado la posibilidad de establecer días y horarios de consulta en la escuela, se han elaborado y entregado módulos y se han desarrollado capacidades para poder hacer llegar a los estudiantes los materiales a través del celular.

Esta capacidad de adaptarse al cambio, involucrando también la transformación, la capacidad de aprendizaje, la innovación, la renovación y la reorganización es mencionada por numerosos autores (Folke, 2006; López Bracamonte y Limón Aguirre, 2017).

En relación con la salud, el barrio Las Heras cuenta con un Centro de Atención Primaria al que asiste la población tanto de Las Heras como de Parque Palermo y también de barrios cercanos. El Centro de Salud posee una fuerte inserción en la comunidad que excede la atención específica. Durante la pandemia, esta atención aparece como uno de los problemas más críticos destacados por los referentes. A esto se suma que las pérdidas de fuentes de trabajo durante 2020 han hecho que la población de los barrios se quede sin servicios sociales, incrementando la demanda.

Por otra parte, una situación crítica es la reducción del personal médico que fue trasladado para atender las demandas de otros barrios y la falta de medicación para enfermedades crónicas no transmisibles como cardiopatías, hipertensión o diabetes. En este contexto, referentes del Centro de Salud señalan que han desarrollado estrategias con otros Centros de la zona para intercambiar medicación en función de las necesidades y generar articulaciones conjuntas para acceder a programas nacionales que les permitan contar con la medicación necesaria para la población. 
Las actividades comunitarias poseen una fuerte impronta en los barrios y están ligadas a talleres culturales desarrollados desde las instituciones y organizaciones barriales en comedores comunitarios, merenderos y sociedades de fomento. Estas actividades se vieron suspendidas en el marco de la pandemia, lo cual ha impactado negativamente en la comunidad. No obstante, los relevamientos y el trabajo con los actores realizados a principios de 2021 revelan una activación mediante la adaptación desde el distanciamiento social, promoviendo su desarrollo al aire libre. En este sentido, los referentes destacan la importancia que ha adquirido el espacio público en el contexto crítico.

Ambos barrios cuentan con espacios destinados a plazas, que constituyen un ámbito de encuentro para la comunidad. La plaza principal del barrio Las Heras está ubicada entre las calles Eduardo Peralta Ramos, Gutemberg, Heguilor y William Morris. Frente a ella se localiza el Centro de Atención Primaria de Salud (CAPS), la Sede de la Asociación Vecinal de Fomento y la escuela primaria (EEP 74) y secundaria (EES 69), ambas provinciales. También en Las Heras, hacia el sector de las vías, se encuentra otra plaza que corresponde al Barrio Dignidad. Frente a ésta, se localiza la Casa de Encuentro Comunitario (CEC), aún en construcción, promovida desde la organización “Cambio de Paso". La plaza de Parque Palermo está ubicada entre las calles Lobería, Manrique, Labardén y Ayelep, sin edificios públicos vinculados con ella.

Las plazas mencionadas cuentan con playones deportivos, veredas y equipamiento (juegos, bancos y cestos), que han otorgado cierta potencialidad de uso de los espacios; sin embargo, al mismo tiempo, han restringido la atención a la identidad propia de cada uno de ellos. Asimismo, el estado de conservación de los equipamientos manifiesta falencias.

Por otra parte, en Parque Palermo, además de la plaza mencionada, se encuentra en la manzana 27 (denominada "El Triángulo", ubicado entre las calles García Lorca, Reservistas y las vías del ferrocarril) un asentamiento dentro del cual un grupo vecinos propone preservar un espacio con fines de uso público. Los vecinos plantean la posibilidad de mejorarlo y conservarlo, resaltando su valor en el contexto de pandemia. En este mismo barrio, existe un área de esparcimiento (cancha de fútbol), ubicada en la intersección entre la calle Ciudad de San Cayetano y la avenida Mario Bravo, que ha adquirido una fuerte dinámica durante 2020 y que actualmente conforma un espacio de socialización sobre el cual se proyectan nuevas iniciativas comunitarias (sector de juegos y huerta).

Los comedores y merenderos existentes continuaron su actividad e incluso surgieron nuevos. Sin embargo, tal como informan los referentes, fue necesario modificar la modalidad implementada, a través de la entrega de viandas a las familias. En el marco planteado y en otra escala que supera a la barrial, surgen los Comités Barriales de Emergencia (CBE) en la ciudad de Mar del Plata y en el partido de General Pueyrredon. Los CBE son dispositivos de articulación comunitaria impulsados por organizaciones sociales con el objetivo de distribuir recursos y redes para sostener y acompañar las medidas asociadas al aislamiento social, preventivo y obligatorio. Situados en diferentes áreas del distrito, los CBE tienen como uno de sus principales objetivos el fortalecimiento de la red de comedores escolares, comunitarios, 
VULNERABILIDAD SOCIOAMBIENTAL Y RESILIENCIA EN DOS BARRIOS DEL BORDE URBANO DE LA CIUDAD DE MAR DEL PLATA: APORTES PARA LA GESTIÓN LOCAL
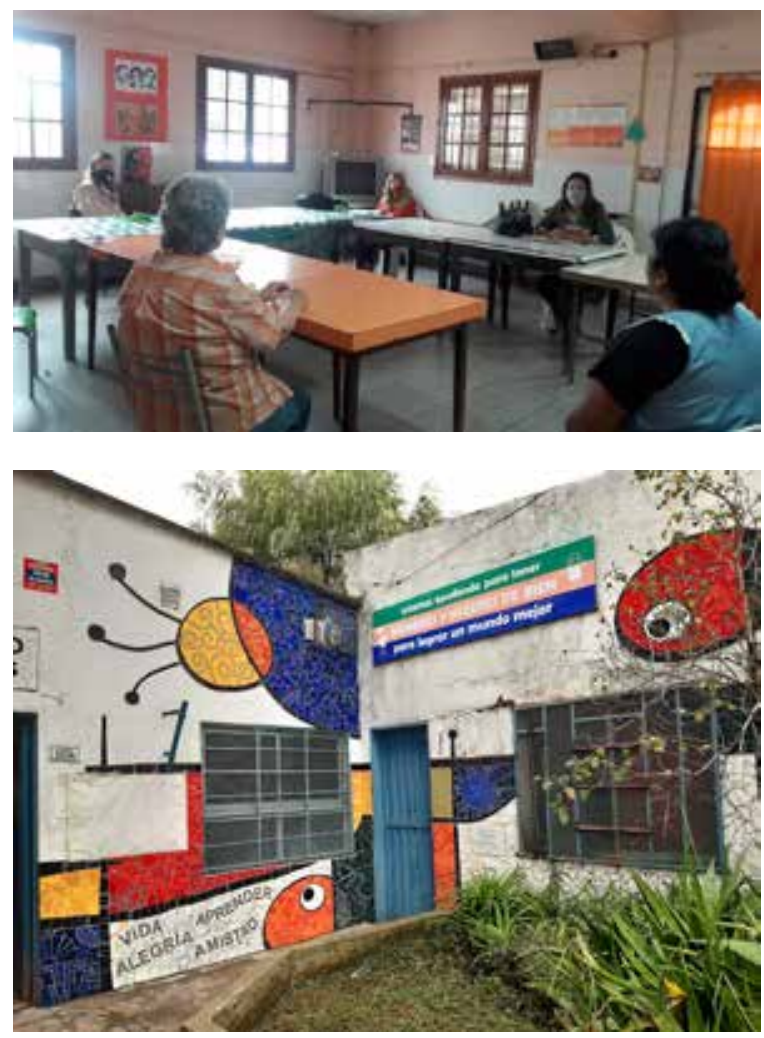

cocinas populares, merenderos, programas de asistencia alimentaria para adultos mayores, etc, para integrarla, coordinarla y abastecerla de insumos de limpieza y protección y de alimentos secos y frescos (CESP, 2020).

Los CBE se articulan con iglesias, clubes, organizaciones políticas, sociedades de fomento, centros y salas de salud, dispositivos estatales de contención, etc, es decir, un amplio espectro de organizaciones intermedias, que en ocasiones convergen a partir de intereses conjuntos, y en otras no. Los barrios Parque Palermo y Las Heras integran la zona suroeste y funcionan dos comités, uno en cada barrio. Independientemente de ello, existen organizaciones de base no ligadas a los $\mathrm{CBE}$ que han fortalecido redes

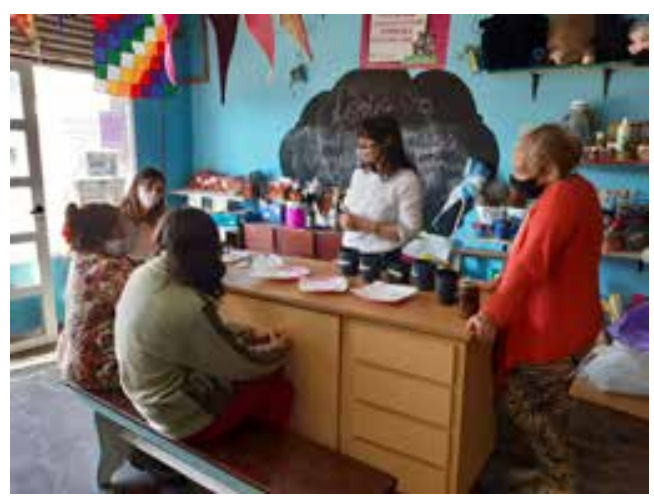

Fig. 4. Barrios Las Heras y Parque Palermo: comedores comunitarios (2021). Fuente: los autores.

para dar respuesta a las necesidades de la comunidad, especialmente alimentarias. Además, en muchas familias se han profundizado conflictos familiares y aquellos vinculados con la violencia de género. En virtud de ello, desde las instituciones y organizaciones se han fortalecido las redes de acompañamiento para sostener a las familias que atraviesan por estas situaciones, a fin de orientarlas en su proceder. Estas redes de promotores funcionan como un nexo entre la comunidad y las instituciones.

En línea con las acciones generadas directamente por los CBE, desde las organizaciones comunitarias comparten y reparten excedentes de alimentos a través de las instituciones para llegar a todos los sectores de los barrios. La Figura 4 muestra algunos comedores existentes en ambos barrios. Por otra parte, y a fin de atender a demandas de alimentos frescos, durante 2020 se incrementaron las huertas familiares en las viviendas a quienes se brindó acompañamiento desde el programa Pro-Huerta del Instituto Nacional de Tecnología Agropecuaria (INTA). 
Asimismo, a través de las instituciones educativas se realizaron los repartos de bolsones de alimentos que provee la provincia de Buenos Aires y también se brinda acompañamiento familiar articulando con otras instituciones del barrio. Los referentes mencionan que durante la pandemia se aportan contenidos que tienen que ver con la higiene y el distanciamiento social.

Entre las fortalezas que mencionan los referentes barriales se destacan las articulaciones interinstitucionales y con organizaciones sociales, los lazos afectivos y la capacidad de trabajo en equipo. Por otra parte, se menciona el deseo e intención permanente de superación personal.

En los términos de Arciniega (2013), muchas de las dificultades que atraviesan las comunidades son vistas como oportunidades. Por otro lado, Suárez Ojeda (2001) afirma que las comunidades resilientes presentan ciertas características específicas a las que se reconoce como pilares de resiliencia comunitaria, entre las que se pueden señalar la autoestima colectiva, que hace referencia al sentido de pertenencia, y la identidad cultural, que representa el conjunto de tradiciones, costumbres y creencias propias de la comunidad.

La discontinuidad de ingresos laborales incidió en la provisión de alimentos en gran parte de los hogares. En este marco, de manera semejante a lo que sucedió en el conurbano bonaerense, se desplegaron un conjunto de estrategias que mostraron tanto la centralidad de la acción estatal como la activación de un rico entramado organizacional a la hora de dar respuesta provisoria a la crisis sociosanitaria, que fue tomando un vigor y nivel de estructuración creciente (Maceira, 2021).
La pandemia, además de visibilizar los problemas, ha permitido evidenciar la capacidad de respuesta de la comunidad de los barrios ante la crisis, tal como lo demuestra el conjunto de acciones mencionadas previamente. Sin embargo, estas adaptaciones y capacidades no se reflejan a nivel urbano, donde aún la ciudad, y especialmente el hábitat de estos barrios "de borde", no mostraron adaptaciones para el desarrollo de las actividades cotidianas. En función de ello, emerge el interés de referir al concepto de resiliencia urbana que, en términos generales, puede definirse como la capacidad que tiene una ciudad de adaptarse a las necesidades y crisis.

\section{RESILIENCIA URBANA, UN ENFOQUE PARA REPENSAR LOS BORDES DE LA CIUDAD}

Como fue adelantado, estas áreas situadas hacia el borde de la ciudad se caracterizan por el crecimiento espontáneo y una frágil estructuración con el resto de la ciudad. Coexisten usos marginales diversos que, por razones económicas o de compatibilidad, no pueden emplazarse dentro del núcleo urbano. Por tratarse de áreas situadas en el perímetro del área urbana, presentan una morfología imprecisa y mutante. Sin embargo, resultan espacios con fuerte dinámica constructiva, esencialmente de carácter informal, con escasa cohesión y, en términos urbanos, con débil capacidad de respuesta ante adversidades. Esta capacidad de respuesta puede analizarse desde la perspectiva de la resiliencia urbana.

En los últimos años, la resiliencia urbana ha surgido como un tema central del desa- 
VULNERABILIDAD SOCIOAMBIENTAL Y RESILIENCIA EN DOS BARRIOS DEL BORDE URBANO DE LA CIUDAD DE MAR DEL PLATA: APORTES PARA LA GESTIÓN LOCAL

rrollo de las ciudades y asentamientos humanos como base para una amplia gama de intervenciones. Es definida como una cualidad del desarrollo urbano sostenible que reconoce el área urbana como un sistema complejo y dinámico que debe adaptarse continuamente a diversos desafíos en una manera integrada y holística a través de su estructura funcional, organizacional, física y espacial, incluyendo diferentes escalas (ONU-Hábitat, 2015).

El Programa de las Naciones Unidas para los Asentamientos Humanos (ONU-Hábitat) propone entre sus objetivos aumentar la resiliencia de las ciudades frente a los impactos de las crisis, como es el caso de la pandemia. Así, pretende aumentar la capacidad de los asentamientos humanos para resistir y recuperarse rápidamente de cualquier peligro, que implica reducir el riesgo y volver a la estabilidad (ONUHábitat, 2017). Sin duda, el COVID-19 ha "desestabilizado" a las ciudades y comunidades, impactando especialmente en los sectores más vulnerables, como son las áreas de borde.

Desde una perspectiva conceptual, en el año 2012 la ONU describió las características que debiera tener una ciudad resiliente: a) un gobierno que busca la urbanización sostenible y destina los recursos necesarios para antes, durante y después de una amenaza; b) viviendas y barrios que cuenten con servicios e infraestructura adecuados que permitan minimizar los efectos de los peligros; c) existencia de información de base sistematizada y compartida; d) capacidad de planificación, acción y participación conjunta en los procesos de toma de decisión; e) desarrollo de sistemas de monitoreo para responder ante los peligros y minimizar los impactos; f) recuperabilidad para reanudar actividades.
Más allá de respuestas aisladas para hacer frente a la emergencia, estas condiciones están lejos de alcanzarse en los barrios analizados. En 2020 se han acelerado procesos de ocupación en diversos sectores en ambos barrios como consecuencia de la crisis. Así, en el barrio Las Heras, ha sido ocupada una manzana completa -ubicada con cierta centralidad-, entre las calles Heguilor, García Lorca, Benito Lynch y Eduardo Peralta Ramos, que se encontraba vacía y en la que se desarrollaban actividades de esparcimiento. Esta manzana ha sido completamente subdividida y ocupada por nuevos habitantes. En el barrio Parque Palermo, el proceso se ha desarrollado en diversas áreas de borde tales como terrenos lindantes con las vías del Ferrocarril, particularmente frente al asentamiento existente en "El Triángulo" (manzana 27). También, la expansión ha tenido lugar sobre la calle Santiago Lacantú, lindante a predios rurales y sobre la calle Labardén, en manzanas más extensas, que se encontraban desocupadas. Por último, se observan nuevos asentamientos dispersos sobre terrenos bajos laterales a la avenida Tetamanti. Estas áreas se muestran en la Figura 5.

En estos sectores, las condiciones habitacionales, la infraestructura y el acceso a los servicios esenciales no cubren las necesidades ni garantizan el derecho a una vivienda adecuada por parte de sus habitantes. La capacidad de planificación y acción para direccionar los procesos de urbanización son débiles, así como la disponibilidad de recursos para garantizar condiciones mínimas de habitabilidad.

En relación con la infraestructura y servicios, desde el ProMeBa, los barrios fueron abastecidos por el sistema de agua potable, desagües pluviales, gas natural, cordón cuneta, 


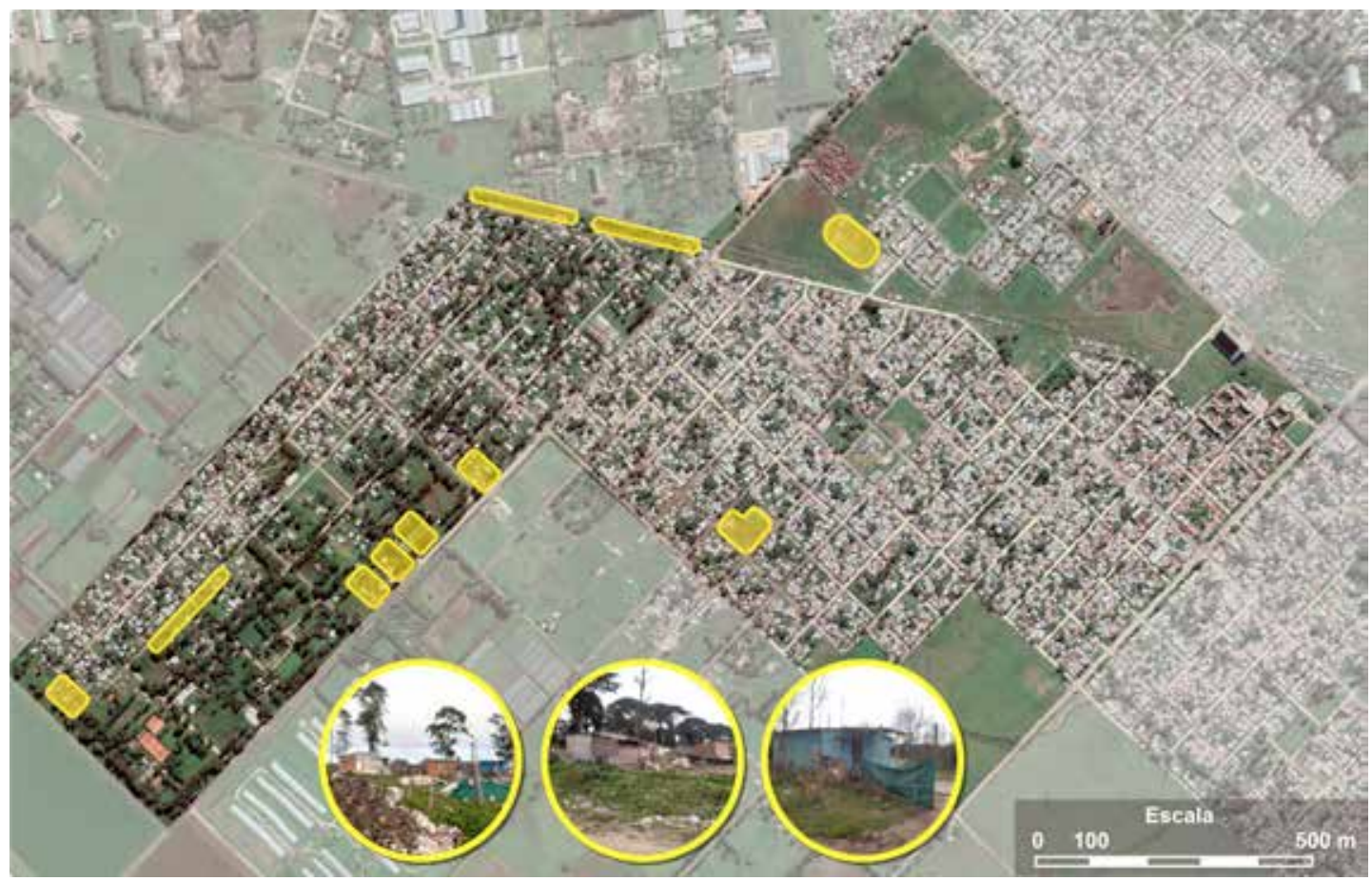

Fig. 5. Barrios Las Heras y Parque Palermo: localización de asentamientos en crecimiento durante 2020. Fuente: Elaboración de los autores sobre la base de Google Earth.

carpeta asfáltica y alumbrado público. Aunque los servicios se extendieron en ambos barrios, esto no significa que efectivamente estén conectados en los hogares. Esto se debe, entre otras cuestiones, a los costos que implica la conexión de los servicios al interior de las viviendas ${ }^{8}$, pero también a las irregularidades en la tenencia de la tierra. En relación con este último aspecto, cabe destacar que ProMeBa cuenta con un componente legal enfocado en la regularización de la tenencia de la tierra.

8 Los costos de conexión de los servicios al interior de las viviendas generalmente son asumidos por los propios usuarios. No obstante, se realizaron en los barrios algunas gestiones con la finalidad de brindar asistencia en este sentido, pero no prosperaron.
Más allá de lo mencionado, un tema pendiente es el estado de las calles y la frecuencia del transporte público. Los referentes de Parque Palermo señalan como problemas prioritarios la frecuencia del transporte público, el estado crítico de las calles y el alumbrado insuficiente que incide en la seguridad. Por su parte, haciendo mención al barrio Las Heras, los referentes plantean la necesidad de mantenimiento de las calles y mencionan también como problema la frecuencia del transporte público.

Otra cuestión importante a destacar son las dificultades de vinculación de estos barrios con otros de la ciudad. En este sentido, el terraplén que sustenta la traza de las vías del Ferrocarril (ramal que se encuentra inactivo desde 
VULNERABILIDAD SOCIOAMBIENTAL Y RESILIENCIA EN DOS BARRIOS DEL BORDE URBANO DE LA CIUDAD DE MAR DEL PLATA: APORTES PARA LA GESTIÓN LOCAL

2013) que atraviesa el Barrio Las Heras, genera un obstáculo físico que sólo admite la conexión en forma segura por las avenidas Tetamanti y Polonia. Existen escasos ejes de jerarquía menor, sin carpeta asfáltica, con escaso mejorado y carentes de mantenimiento, por los que es muy difícil acceder a ese sector.

Las limitaciones en el acceso a infraestructura, servicios y vivienda adecuada ponen de manifiesto las desigualdades que se intensifican en el contexto de pandemia, especialmente ligadas al surgimiento y expansión de asentamientos informales, incidiendo en la resiliencia urbana.

En función de lo expuesto, la resiliencia urbana estará determinada por la capacidad de respuesta positiva de las ciudades para organizarse y generar estrategias para prevenir $y$ mitigar y adaptarse ante la adversidad. Al respecto, Simonovic y Peck (2013) sostienen que es necesario centrarse en la planificación de la resiliencia urbana con acciones tendientes a fortalecer la capacidad local, incluyendo la participación de la comunidad. Ello permitirá una mejor comprensión de la diversidad, necesidades, fortalezas y vulnerabilidades dentro de las comunidades, ya que las amenazas no afectan a todos de la misma manera.

A partir de lo anterior, se desprende la importancia de articular los procesos de resiliencia comunitaria y urbana para dar respuesta a los problemas emergentes o acentuados en el marco de la pandemia y repensar estrategias de gestión local en un escenario pospandemia.

\section{REFLEXIONES FINALES}

La evaluación de la vulnerabilidad socioambiental revela situaciones críticas, especialmente en Parque Palermo. De esta manera, el análisis de la distribución de IVSA permite establecer los sectores de los barrios que manifiestan condiciones más desfavorables en virtud de los indicadores analizados. La distribución espacial del índice muestra correspondencias entre los resultados obtenidos para los valores extremos de los indicadores considerados en las distintas unidades espaciales. A su vez, se observan fuertes correspondencias con el indicador de NBI. Estas diferencias, verificadas entre los barrios a través del IVSA, no se evidencian cuando se aplica el concepto de resiliencia comunitaria. La resiliencia comunitaria, vista como la capacidad para adaptarse continuamente a las fluctuaciones ambientales, está determinada por la capacidad de respuesta positiva de las comunidades para organizarse ante la adversidad. En este sentido, las comunidades de ambos barrios han demostrado capacidad de adaptación en el marco de la pandemia. Sin embargo, esta capacidad de adaptación no se ha reflejado en la gestión urbana.

En línea con lo señalado por Salimi y AlGhamdi (2020), los resultados obtenidos permiten destacar que la resiliencia urbana conforma un enfoque clave para reducir la vulnerabilidad a largo plazo. Así, las características de la resiliencia urbana debieran considerarse en la fase de planificación de los proyectos urbanos y no cuando ocurren los problemas, como aquellos que se han profundizado durante 2020 en los barrios analizados.

A partir de lo anterior, el estudio realizado en ambos barrios demuestra la necesidad de 
centrarse en fortalecer la resiliencia urbana con acciones tendientes a potenciar la capacidad local, jugando un rol protagónico en este proceso la participación de la comunidad. Esto permitirá una mejor comprensión de la diversidad, las necesidades, las fortalezas y las vulnerabilidades de las comunidades, ya que las amenazas no afectan a todos de la misma manera. Desde esta perspectiva y retomando a Simonovic y Peck (2013), una ciudad resiliente implica una red sostenible de sistemas físicos (construidos y naturales) y comunidades humanas (sociales e institucionales) que poseen la capacidad de sobrevivir, afrontar, recuperarse, aprender y transformarse, a partir de las perturbaciones que surgen de las amenazas, impactos y problemas urbanos.

En función de los resultados obtenidos y sobre la base del concepto de resiliencia urbana, se reflexiona acerca del desafío de avanzar con estrategias que permitan dar respuesta las problemáticas generadas y/o visibilizadas en el contexto de pandemia. En ese sentido, se considera especialmente importante en el corto plazo atender las demandas sanitarias y educativas de la comunidad de ambos barrios, fortaleciendo las articulaciones entre instituciones, organismos de gobierno y organizaciones para responder a los problemas socioeconómicos que inciden en la capacidad de respuesta de las familias. En el corto-mediano plazo, se plantea el desafío de fortalecer la identidad, equipar y mejorar los espacios públicos y comunitarios, los cuales han adquirido un rol fundamental en el contexto de pandemia, asumiendo siempre un enfoque participativo. A su vez, adecuar las infraestructuras a las necesidades emergentes y potenciar la articulación de ambos barrios con la ciudad, contribuirían con objetivos planteados desde la perspectiva de la resiliencia urbana a mediano-largo plazo.

Profundizar en el estudio de las estrategias implementadas por estas comunidades para enfrentar la crisis actual conforma el punto de partida para abordar la resiliencia comunitaria y urbana. Estas estrategias permitirían dar respuesta a las problemáticas emergentes en pos de alcanzar la sostenibilidad de las ciudades. Por otra parte, se enfatiza en la importancia de generar y consolidar estrategias de gestión colaborativa del espacio público a partir de acciones concretas promovidas en conjunto con la comunidad de ambos barrios. Dichas acciones ya se han iniciado. En este marco, la resiliencia comunitaria y urbana, repensadas en términos barriales, constituyen el eje central de una gestión local sostenible en el escenario de la pospandemia.

\section{AGRADECIMIENTOS}

Agradecemos a los equipos del Programa de Mejoramiento de Barrios del Ministerio de Desarrollo Territorial y Hábitat de la Nación Argentina y del Municipio de General Pueyrredon por el acompañamiento permanente en las actividades realizadas. 
VULNERABILIDAD SOCIOAMBIENTAL Y RESILIENCIA EN DOS BARRIOS DEL BORDE URBANO DE LA CIUDAD DE MAR DEL PLATA: APORTES PARA LA GESTIÓN LOCAL

\section{REFERENCIAS}

Arciniega, Juan de Dios. 2013. “La perspectiva comunitaria de la resiliencia”. Psicología política 47: 7-18.

Asadzadeh, Asad; Kötter, Theo; Salehi, Pourya. y Birkmann, Joern. 2017.“Operationalizing a concept: The systematic review of composite indicator building for measuring community disaster resilience". International Journal of Disaster Risk Reduction. 25: 147-162.

Baeza Rodriguez, Manuel Antonio. 2002. De las metodologías cualitativas en investigación científico social. Diseño y uso de instrumentos en la producción de sentido. Concepción: Editorial de la Universidad de Concepción.

Beck, Ulrich. 1998. La sociedad del riesgo. Hacia una nueva modernidad. Barcelona: Paidós.

Brooks, Nick; Aure, Eunica. y Whiteside, Martin. 2014. Assessing the impact of ICF programmes on household and community resilience to climate variability and climate change. Draft Report. UK: Evidence on Demand.

Buzai, Gustavo. 2003. Mapas sociales urbanos. Buenos Aires: Lugar Editorial.

Buzai, Gustavo y Baxendale, Claudia. 2006. Análisis Socioespacial con Sistemas de Información Geográfica. GEPAMA, Buenos Aires: Lugar Editorial.

Cabral, Victoria y Zulaica, Laura. 2015. “Análisis de la vulnerabilidad socioambiental en áreas del periurbano de Mar del Plata (Argentina) expuestas a agroquímicos". Multiciencias, 15, 2: 172-180.
Castro-Díaz, Ricardo. 2013. "Implicancias de la Resiliencia Espacial en la prestación de servicios ambientales en cuencas norandinas". Contribuciones Geográficas, 25: 71-87.

CESP. Impacto territorial de las políticas de articulación local implementadas en el contexto del COVID19 en barrios populares del Partido de General Pueyrredon: capacidad de respuesta a las necesidades emergentes y propuestas para su fortalecimiento [2020]. Primer Informe. Mar del Plata: Centro de Estudios Sociales y Políticos, Facultad de Humanidades, Universidad Nacional de Mar del Plata.

Daga, Daiana; Zulaica, Laura y Vazquez, Patricia. 2015. "Evaluación de la vulnerabilidad socio-ambiental del periurbano de Mar del Plata”. Estudios Socioterritoriales, 18: 45-59.

Ellis, B. Heidi y Abdi, Saida. 2017. "Building community resilience to violent extremism through genuine partnerships". American Psychologist. 72, 3: 289.

Folke, Carl. 2006. "Resilience: The emergence of a perspective for social-ecological systems analyses". Global environmental change. 16, 3: 253-267.

Garay, Alfredo. 2000. Gestión ambiental de infraestructura y servicios urbanos. Mar del Plata: GADU-FAUD-UNMdP.

García de León, Armando. 1997. “Empleo de una metodología multivariada para la clasificación de unidades territoriales". 
Revista del Colegio Mexicano de

Geografía, 14: 5-20.

Giraud-Herrera, Loraine Mayrim y Morantes-

Quintana, Giobertti Raul. 2017.

"Aplicación del análisis multivariante para la sostenibilidad ambiental urbana".

Bitácora. 27, 1: 89-100.

Hallegatte, Stephan y Engle, Nathan L. 2019.

"The search for the perfect indicator:

Reflections on monitoring and evaluation

of resilience for improved climate risk management". Climate Risk Management. 23: 1-6.

INDEC. 2010. Censo Nacional de Población, Hogares y Viviendas. Instituto Nacional de Estadísticas y Censos. Buenos Aires.

INDEC. 2015. Estimaciones de población por sexo, departamento y año calendario 2010 - 2025. Nº 38 Serie de Análisis Demográfico. Instituto Nacional de Estadísticas y Censos. Buenos Aires.

INHUS. 2020. Proyecto Monitoreo y seguimiento de las estrategias para minimizar la circulación del COVID_19 en el Municipio de General Pueyrredon. Primer Informe técnico. Mar del Plata: Instituto de Humanidades y Ciencias Sociales, CONICET, Universidad Nacional de Mar del Plata.

Joerin, Jonas; Shaw, Rajib; Takeuchi, Yukiko y Krishnamurthy, Ramasamy. 2012. "Assessing community resilience to climate-related disasters in Chennai, India”. International Journal of Disaster Risk Reduction. 1: 44-54.
Lavell, Allan. 1996. "Degradación Ambiental, Riesgo y Desastre Urbano. Problemas y Conceptos: Hacia la Definición de una Agenda de Investigación”. En Ciudades en riesgo. Degradación Ambiental, Riesgos urbanos y Desastres en América Latina, editado por María Augusta Fernández, 2159. Perú: Red de Estudios en Prevención de desastres en América Latina.

Leong, Ching. 2016. "Resilience to climate change events: The paradox of water (In)security". Sustainable Cities and Society. 27: 439-447.

López Bracamonte, Fabiola. M. y Limón Aguirre, Fernando. 2017. “Componentes del proceso de resiliencia comunitaria: conocimientos culturales, capacidades sociales y estrategias organizativas". PSIENCIA. Revista Latinoamericana de Ciencia Psicológica. 9: 1-13.

Maceira, Verónica. 2021. "Condiciones de vida de los hogares y estrategias de los actores". En El conurbano bonaerense en pandemia Alcances y desafíos desde una perspectiva multidimensional, compilado por Rodrigo Carmona, 17-42. Los Polvorines: Universidad Nacional de General Sarmiento.

Maguire, Brigit y Cartwright, Sophie. 2008. Assessing a community's capacity to manage change: a resilience approach to social assessment. Canberra: Bureau of Rural Sciences.

Mignaqui, Iliana. 2012. "Planes y proyectos territoriales. Escenarios de la metrópolis planificada". Revista Iberoamericana de Urbanismo. 8: 121-137 
VULNERABILIDAD SOCIOAMBIENTAL Y RESILIENCIA EN DOS BARRIOS DEL BORDE URBANO DE LA CIUDAD DE MAR DEL PLATA: APORTES PARA LA GESTIÓN LOCAL

Natenzon, Claudia. 1998. "Riesgo, vulnerabilidad e incertidumbre. Desastres por inundaciones en Argentina". En: Seminario sobre Problemas ambientales e vulnerabilidade. Abordagens integradoras para o campo da Saude Publica. Ministerio de la Salud. Río de Janeiro, pp. 1-19.

Natenzon, Claudia y Besalú Parkinson. 2020. El derecho como instrumento de adaptación al cambio climático. Revisión de sentencias relativas a desastres por inundaciones urbanas. AREA. 26, 1: 1-12.

ONU-Hábitat. 2015. Resiliencia urbana, Conferencia de las Naciones Unidas sobre la Vivienda y el Desarrollo Urbano Sostenible. Nueva York: Organización de Naciones Unidas.

ONU-Hábitat. 2017. Temas urbanos: Resiliencia. Disponible en: https://unhabitat.org/es/ node/3774

Pírez, Pedro. 2016. "Las heterogeneidades en la producción de la urbanización y los servicios urbanos en América Latina". Territorios 34: 87-112

Salimi, Mohsen y Al-Ghamdi, Sami. 2000. "Climate change impacts on critical urban infrastructure and urban resiliency strategies for the Middle East". Sustainable Cities and Society. 54: 1-17.

Sánchez González, Diego y Egea Jiménez, Carmen. 2011. "Enfoque de vulnerabilidad social para investigar las desventajas socioambientales. Su aplicación en el estudio de los adultos mayores". Papeles de Población, 17, 69: 151-185.
Salvia, Agustín; Bonfiglio, Juan Ignacio y Robles. 2021. Efectos de la pandemia COVID-19 sobre la dinámica del bienestar en la Argentina urbana. Una mirada multidimensional acerca del impacto heterogéneo de la crisis tras una década de estancamiento económico (2010-2020). Documento Estadístico - Barómetro de la Deuda Social Argentina. Ciudad Autónoma de Buenos Aires: Educa.

Scherzer, Sabrina; Lujala, Päivi y Rød Jan Ketil. 2019. "A community resilience index for Norway: An adaptation of the Baseline Resilience Indicators for Communities (BRIC)". International Journal of Disaster Risk Reduction 36: 101107.

Schuschny, Andrés y Soto, Humberto. 2009. Guía metodológica, diseño de indicadores compuestos de desarrollo sostenible. Santiago de Chile: CEPAL, Naciones Unidas.

Simonovic, Slobodan. y Peck, Angela. 2013. Dynamic Resilience to Climate Change Caused Natural Disasters in Coastal Megacities Quantification Framework. British Journal of Environment \& Climate Change. 3, 3: 378-401.

Suárez Ojeda, Elvio Nestor. 2001. Resiliencia descubriendo las propias fortalezas. Buenos Aires: Paidós.

Tonon, Graciela. 2015. Relevance of the Use of Qualitative Methods in the Study of Quality of Life. In G. Tonon (Ed.), Qualitative Studies in Quality of Life Methodology and Practice. Social indicators research series. Vol. 55, Heilderberg, London, New York Springer: Dordretch, pp. 3-21. 
UNISDR. 2017. Terminología para el marco de la Reducción del Riesgo de Desastres. Oficina de las Naciones Unidas para la Reducción del Riesgo de Desastres. Disponible en: http://www.unisdr.org/we/ inform/terminolog

Visintini, María Laura y Castro-Díaz, Ricardo. 2018. "Aplicación del índice de vulnerabilidad social en el estudio de riesgos de desastre en la costa santafesina" [2018]. En: Jornadas Platenses de Geografía y XX Jornadas de Investigación y de Enseñanza en Geografía. FaHCE, Universidad Nacional de La Plata. La Plata, pp. 1-20.

Wilches Chaux, Gustavo. 1993. "La Vulnerabilidad Global". En: Los desastres no son naturales, compilado por Andreu Maskrey, pp. 11-44. Bogotá: Red de Estudios Sociales en Prevención de Desastres en América Latina

\section{MIGUEL RÓTOLO}

Magister Scientiae en Gestión Ambiental del Desarrollo Urbano, Arquitecto y Doctorando en Arquitectura y Urbanismo. FAUD, UNMdP. Profesor Adjunto Ordinario en el Taller Vertical de Diseño Arquitectónico I-III “M”, FAUD, UNMdP. Miembro del equipo de investigación en el Proyecto Indicadores de Sustentabilidad Urbana y Ambiental: Aplicaciones en el Partido de General Pueyrredon y en el Centro-Sur Bonaerense, dirigido por la Dra. Lic. Laura $\mathrm{Zu}-$ laica en el ámbito del Instituto del Hábitat y el
Wilson, Geoff A. 2013. "Community resilience, policy corridors and the policy challenge". Land use policy. 31: 298-310.

Wynne, Brian. 1992. "Uncertainty and environmental learning. Reconceiving science and policy in the preventive paradigm". Global Environmental Change. 2, 2: 111-127.

Zulaica, Laura y Ferraro, Rosana. 2010. "Vulnerabilidad socio-ambiental y dimensiones de la sustentabilidad en un sector del periurbano marplatense". Estudios Socioterritoriales. 8: 197-219.

Zulaica, Laura y Ferraro, Rosana. 2016.

"Vulnerabilidad socioambiental en Monte Caseros, Corrientes: aportes al estudio de las problemáticas socio-espaciales de la localidad", Journal de Ciencias Sociales. 7: 93-114.

Zulaica, Laura y Rampoldi Aguilar, Romina. 2009. "Habitabilidad y calidad de vida en tres barrios del límite urbano-rural de la ciudad de Mar del Plata”. Hologramática. 10, 1: 27-58.

Ambiente, FAUD, UNMdP. Subsecretario Académico de la FAUD, UNMdP. Consultor Urbano del Programa Mejoramiento de Barrios (BID), Ministerio de Desarrollo Territorial y Hábitat - UEM General Pueyrredon desde 2013. Ha actuado como Consultor Coordinador del equipo interdisciplinario del Plan Maestro Corredor Verde Multifuncional - Mar del Plata - Dirección Nacional de Preinversión Municipal (DINAPREM) en 2017-18 y Miembro del Equipo Técnico del Plan Estratégico Mar del Plata en 2006 y 2007. 
VULNERABILIDAD SOCIOAMBIENTAL Y RESILIENCIA EN DOS BARRIOS DEL BORDE URBANO DE LA CIUDAD DE MAR DEL PLATA: APORTES PARA LA GESTIÓN LOCAL

\section{LAURA ZULAICA}

Doctora en Geografía, Magister y Especialista en Gestión Ambiental, Licenciada en Diagnóstico y Gestión Ambiental. Investigadora Adjunta del CONICET, con lugar de trabajo en el Instituto del Hábitat y del Ambiente, FAUD, UNMdP. Profesora Adjunta en la FAUD y en la FH de la UNMdP. Profesora de cursos de posgrado en distintos ámbitos académicos. Directora y codirectora de proyectos de investigación radicados en la UNMdP y de becarios y tesistas de grado y posgrado. Se destaca su participación en proyectos interdisciplinarios. Es Consultora Ambiental del Programa de Mejoramiento de Barrios (BID), Ministerio de Desarrollo Territorial y Hábitat - UEM General Pueyrredon desde 2020. Actualmente, sus investigaciones se centran en la construcción y evaluación de indicadores de sustentabilidad ambiental, entre cuyos campos de aplicación se destacan: gestión ambiental y del hábitat, ordenamiento territorial y evaluación ambiental.
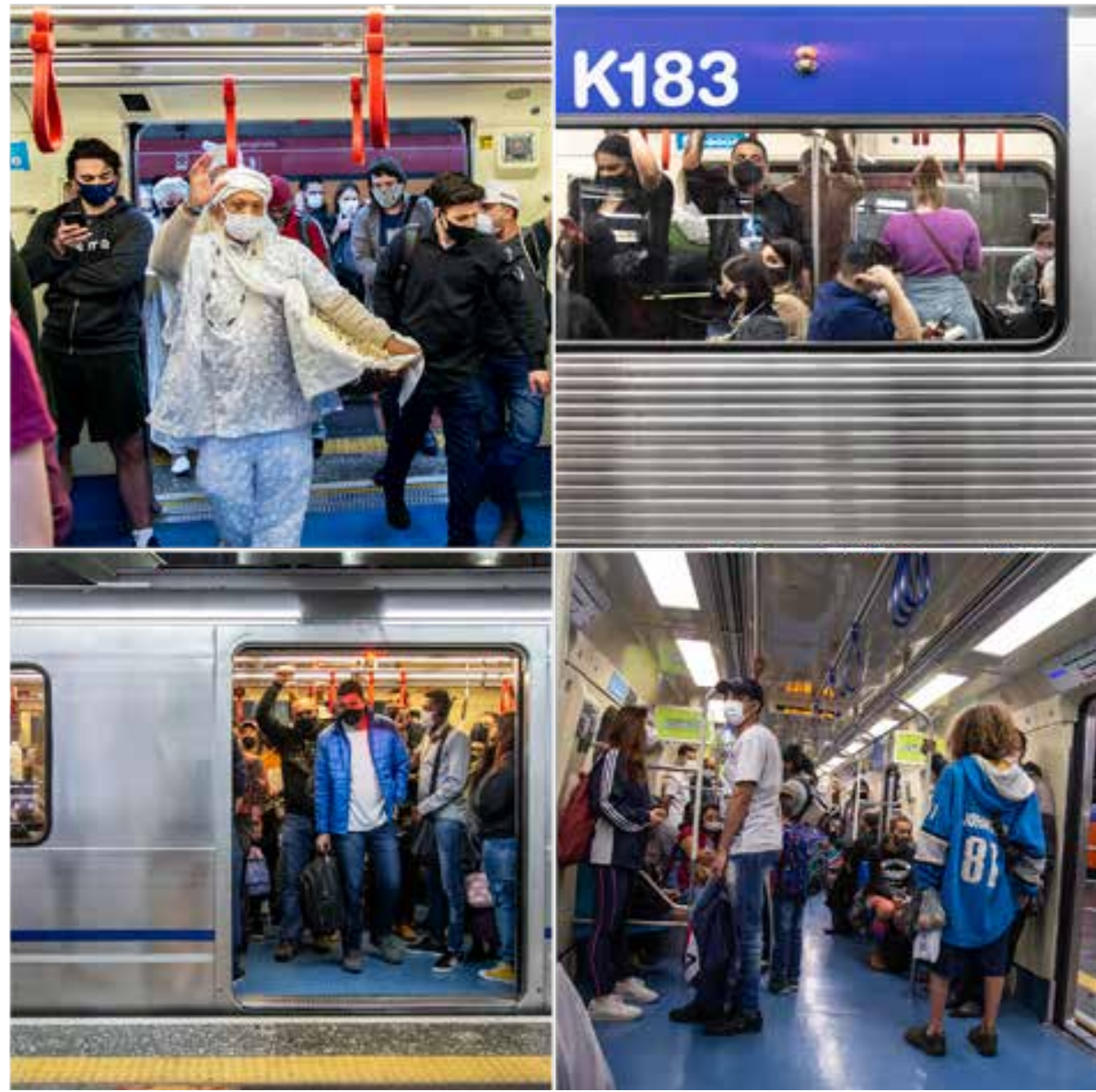